\title{
YOUNG-MEASURE APPROXIMATIONS FOR ELASTODYNAMICS WITH NON-MONOTONE STRESS-STRAIN RELATIONS
}

\author{
Carsten Carstensen $^{1}$ And Marc Oliver Rieger ${ }^{2}$
}

\begin{abstract}
Microstructures in phase-transitions of alloys are modeled by the energy minimization of a nonconvex energy density $\phi$. Their time-evolution leads to a nonlinear wave equation $u_{t t}=\operatorname{div} S(D u)$ with the non-monotone stress-strain relation $S=D \phi$ plus proper boundary and initial conditions. This hyperbolic-elliptic initial-boundary value problem of changing types allows, in general, solely Youngmeasure solutions. This paper introduces a fully-numerical time-space discretization of this equation in a corresponding very weak sense. It is shown that discrete solutions exist and generate weakly convergent subsequences whose limit is a Young-measure solution. Numerical examples in one space dimension illustrate the time-evolving phase transitions and microstructures of a nonlinearly vibrating string.
\end{abstract}

Mathematics Subject Classification. 35G25, 47J35, 65P25.

Received: November 2, 2003.

\section{INTRODUCTION}

The numerical simulation of a nonlinear wave equation

$$
u_{t t}=\operatorname{div} S(D u) \quad \text { in } Q=\Omega \times(0, T),
$$

the space-time domain, plus boundary and initial conditions is certainly one of the more important tasks in computational sciences and engineering. The hyperbolic nature of (1.1) possibly leads to discontinuities (e.g. shocks). The nonlinear stress-strain relation $S=D \phi$ is modeled by the gradient of a smooth function $\phi$. Even for convex $\phi$ it is, in general, unknown whether or not there always exist weak solutions in the class of Sobolev functions. There are, however, affirmative results if $\Omega \subset \mathbb{R}$ is one-dimensional, in particular the seminal works by DiPerna, Murat and Tartar where Young-measures had been used to obtain global existence results (see, e.g., [27] for further references). In higher dimensions there are local results for small $T>0$ [11].

The simulation of phase-transition problems motivates nonconvex energy densities $\phi$ where weak solutions may not exist. Instead, oscillations are likely to occur which can be described mathematically using the concept

\footnotetext{
Keywords and phrases. Non-monotone evolution, nonlinear elastodynamics, Young-measure approximation, nonlinear wave equation.

1 Department of Mathematics, Humboldt-Universität zu Berlin, Unter den Linden 6, 10099 Berlin, Germany.

e-mail: cc@math.hu-berlin.de

2 Scuola Normale Superiore, Piazza dei Cavalieri 7, 56100 Pisa, Italy. e-mail: rieger@sns .it
} 
of Young-measure valued solutions (see Sect. 2). The following example suggests that even if classical solutions are present, there may exist further Young-measure solutions with more physical relevance.

Example. Let $\Omega=(0,1)$ denote the unit interval and let $\phi(y)=\left(1-y^{2}\right)^{2}$ denote the 2 -well energy density. Given a parameter $\lambda \in \mathbb{R}$, the initial conditions $u_{0}(x)=\lambda x$ and $u_{1}(x)=0$ for $0<x<1$ and the boundary conditions $u(x, \cdot)=\lambda x$ for $x=0$ and $x=1$ we obtain the classical stationary solution $u(\cdot, t)=u_{0}(\cdot)$. For $-1<\lambda<1$ and $\mu:=(1-\lambda) / 2$, the measure $\nu:=\mu \delta_{-1}+(1-\mu) \delta_{+1}$ defines a Young-measure solution in the sense specified below in this paper. Here and below, $\delta_{ \pm 1}$ denotes the Dirac measure supported at \pm 1 , the two wells where the energy density $\phi$ is minimal.

In contrast to the smooth classical solution $u_{0}$, the measure valued solution defined by $\nu$ does describe oscillations in agreement to observations in physical experiments. Moreover, the total energy of the Youngmeasure solution is zero, whereas the classical solution has the energy $\phi(\lambda)>0$. Finally, for $|\lambda|<3^{-1 / 2}$, the classical solution is instable, a linearized model leads to an oscillator with a spring of negative stiffness $\phi^{\prime \prime}(\lambda)$. However, the Young-measure solution is stable, in the sense that it corresponds to a state with minimal energy.

In conclusion, the Young-measure solution is a very relevant object; its numerical simulation appears an innovative task and is therefore introduced in this paper.

For the numerical simulation with (1.1), a viscous regularization

$$
u_{t t}=\operatorname{div} S(D u)+\mu \Delta u_{t}
$$

has been proposed $[7,16]$ for a small parameter $\mu>0$, the viscosity. It is known that the initial-boundary value problem with (1.2) has a solution if $S$ is, e.g., globally Lipschitz continuous, but possibly non-monotone [14]. Moreover, the numerical analysis of a backward time-step discretization combined with a finite element discretization of $\Omega$ in space was reported in [16] to be instable for $\mu>0$ and a nonconforming FEM was suggested. Recently, the convergence for the conforming FEM was established under strong regularity assumptions of the unique exact solution in [7]. The situation for $\mu=0$, however, cannot be handled since some a priori bounds of the discrete FE solutions are missing in the passage to the limit. As a consequence, measure-valued solution concepts are necessary which are weak enough to allow high oscillations in the limit. In particular the notion of Young-measures as they were originally introduced by L.C. Young under the name "generalized curves" (see $[28,29]$ ) turned out to be an adequate concept in the 1970s with the work of Tartar (see [26] and [1,3] for stimulating contributions).

The numerical simulation of related nonconvex minimization problems has been studied in $[8-10,17]$ for a direct minimization, in $[4,5]$ for a convexified situation, and in $[4,6,19,24]$ for a measure-valued generalization. Time-evolution problems have been studied analytically in $[12,13,15,21-23,25]$ and are numerically addressed in this paper in the spirit of [6].

The remaining part of the paper is organized as follows. Section 2 establishes the concept of Young-measure solutions of (1.1) and states an existence result in Theorem 2.1. The consistent discretization in space-time is addressed in Section 3 where we introduce a numerical scheme, prove existence of discrete solutions, a discrete energy estimate, and a convergence result. The numerical realization is explained in Section 4 before several numerical simulations are reported in Section 5. Some extending remarks conclude the paper with Section 6.

We will use the following notations: throughout this article, $\Omega$ denotes an open domain in $\mathbb{R}^{n}$ with Lipschitz boundary. $L^{p}(\Omega)$ denotes the Lebesgue space of functions $u$ such that $|u|^{p}$ is integrable; $L^{\infty}(\Omega)$ the space of bounded functions; $W^{m, p}(A, B)$ is the Sobolev space of all functions $u: A \rightarrow B$ with $D^{m} u$ in $L^{p}(\Omega)$ for $p \in[1, \infty]$, where $D^{m}$ denotes partial derivatives of order $m \in \mathbb{N}$. We write $H^{m}(A, B):=W^{m, 2}(A, B)$.

Let $p<\infty$. A sequence of functions $u_{n}$ is said to be weakly converging to a limit function $u$ in $L^{p}(\Omega)$ if $u_{n} \in L^{p}(\Omega)$ and for all test functions $\zeta \in L^{p^{\prime}}$ with $1 / p+1 / p^{\prime}=1$ we have

$$
\int_{\Omega} u_{n} \zeta \rightarrow \int_{\Omega} u \zeta
$$


We denote this by $u_{n} \rightarrow u$ in $L^{p}(\Omega)$. Similarly we say that $u_{n} \rightarrow u$ in $W^{m, p}(\Omega)$ if $u_{n} \in W^{m, p}(\Omega)$ and for all $\zeta \in W^{m, p^{\prime}}(\Omega)$

$$
\int_{\Omega} u_{n} D^{m} \zeta \rightarrow \int_{\Omega} u D^{m} \zeta
$$

A function $f: \mathbb{R}^{m \times n} \rightarrow \mathbb{R}$ is called quasiconvex if it satisfies

$$
\int_{\Omega} f(P+D \phi) \mathrm{d} x \geq|\Omega| f(P)
$$

for every test function $\phi$ (i.e. $\phi \in C^{\infty}(\Omega)$ with supp $\phi$ compactly in $\Omega$ ) and every $P \in \mathbb{R}^{m \times n}$.

Finally by $\phi^{* *}$ we denote the convexification of $\phi$ (the largest convex function below $\phi$ ) and by $\phi^{q c}$ the quasiconvexification of $\phi$ (the largest quasiconvex function below $\phi$ ).

\section{YOUNG-MEASURE-VALUED SOLUTIONS}

The aim of this section is to establish a time discretization scheme for nonconvex elastodynamical equations converging to a Young-measure solution. The statistics of the oscillating deformation gradient of our approximate solution is described by a probability measure. The constructive existence proof in Section 3 is the basis for the numerical simulations below.

Given initial data $u_{0} \in H_{0}^{1}\left(\Omega ; \mathbb{R}^{n}\right), v_{0} \in L^{2}\left(\Omega ; \mathbb{R}^{n}\right)$, and boundary data $g \in H^{1}(\Omega)$, the nonlinear system of elastic time evolution reads

$$
\begin{aligned}
u_{t t} & =\operatorname{div} S(D u) \quad \text { (in the sense of distributions), } \\
u(x, 0) & =u_{0}(x) \quad \text { for almost every } x \in \Omega \\
u_{t}(x, 0) & =v_{0}(x) \quad \text { for almost every } x \in \Omega \\
u(x, 0) & =g(x) \quad \text { for } x \in \partial \Omega \text { (in the sense of traces). }
\end{aligned}
$$

The non-negative energy density $\phi \in \mathcal{C}^{1}\left(\mathbb{R}^{m \times n}\right)$ and its derivative $S:=D \phi: \mathbb{R}^{m \times n} \rightarrow \mathbb{R}^{m \times n}$ satisfy for some constants $\alpha, \beta>0$ certain growth conditions formulated with the spaces $\mathcal{E}_{0}$ and $\mathcal{F}_{0}$.

By $\mathcal{E}_{0}$ we denote the space of continuous functions with specified quadratic growth and by $\mathcal{F}_{0}$ the space with linear growth,

$$
\begin{aligned}
& \mathcal{E}_{0}=\left\{f \in \mathcal{C}\left(\mathbb{R}^{m \times n} ; \mathbb{R}_{+}\right) \mid \lim _{\|A\| \rightarrow \infty} \frac{f(A)}{1+\|A\|^{2}} \text { exists and is finite }\right\}, \\
& \mathcal{F}_{0}=\left\{f \in \mathcal{C}\left(\mathbb{R}^{m \times n} ; \mathbb{R}_{+}\right) \mid \lim _{\|A\| \rightarrow \infty} \frac{f(A)}{1+\|A\|} \text { exists and is finite }\right\} .
\end{aligned}
$$

The vector spaces $\mathcal{E}_{0}$ and $\mathcal{F}_{0}$ are endowed with the norms

$$
\|f\|_{\mathcal{E}_{0}}:=\sup _{A} \frac{|f(A)|}{1+\|A\|^{2}} \quad \text { and } \quad\|f\|_{\mathcal{F}_{0}}:=\sup _{A} \frac{|f(A)|}{1+\|A\|},
$$

respectively.

A probability measure $\nu$ is a non-negative Radon measure on a set $E$ with $\nu(E)=1$. A Young-measure (or parameterized measure) is a family of probability measures $\left(\nu_{x}\right)_{x \in \Omega}$ on $\mathbb{R}^{N}$ associated with a sequence of measurable functions $\left(f_{j}\right)_{j \in \mathbb{N}}$ with $f_{j}: \Omega \subset \mathbb{R}^{n} \rightarrow \mathbb{R}^{N}$ such that for any continuous function $\phi: \mathbb{R}^{N} \rightarrow \mathbb{R}$ the function

$$
\bar{\phi}(x)=\int_{\mathbb{R}^{N}} \phi(F) \mathrm{d} \nu_{x}(F)=:\left\langle\nu_{x}, \phi\right\rangle
$$


is measurable, and for every weakly-converging sequence $\left(f_{j}\right)$ we have for all open subsets $\Omega_{0} \subset \Omega$

$$
\int_{\Omega_{0}}\left(\phi\left(f_{j}\right)\right)_{j}(x) \mathrm{d} x \rightarrow \int_{\Omega_{0}} \bar{\phi}(x) \mathrm{d} x .
$$

We can think of the Young-measure as a "one-point statistic" for the sequence $f_{j}$, i.e. $\nu_{x}$ describes (in a certain sense which can be made mathematically precise [20]) the probability distribution of the values of the sequence $f_{j}$ near $x \in \Omega$. For an introduction into Young measures and their applications see [18,20].

Theorem 2.1 (Existence of Young-measure solution). Given $\phi \in \mathcal{E}_{0}$ with $S:=D \phi \in \mathcal{F}_{0}$, such that $\phi^{q c}$ is convex and $\phi(A) \geq \alpha\left(\|A\|^{2}-1\right), u_{0} \in H^{1}(\Omega), u_{1} \in H^{1}(\Omega), g \in H^{1}(\Omega)$ and $Q:=\Omega \times(0, T) \subset \mathbb{R}^{n+1}$, there exists a Young measure solution $(u, \nu)$ of (2.1)-(2.4) in the sense that

$$
\begin{gathered}
u-g \in W^{1, \infty}\left((0, T) ; L^{2}(\Omega)\right) \cap L^{\infty}\left((0, T) ; W_{0}^{1,2}(\Omega)\right), \\
u(x, 0)=u_{0}(x), \quad u_{t}(x, 0)=u_{1}(x) \text { for all } x \in \Omega, \\
\nu=\left(\nu_{x, t}:(x, t) \in Q\right) \text { is a family of probability measure, } \\
\int_{0}^{T} \int_{\Omega}\left(\langle\nu, S\rangle \nabla \zeta-u_{t} \zeta_{t}\right) \mathrm{d} x \mathrm{~d} t=0 \quad \text { for all } \zeta \in \mathcal{C}_{0}^{\infty}(Q), \\
D u(x, t)=\left\langle\nu_{x, t}, \mathrm{Id}\right\rangle \quad \text { for almost every }(x, t) \in Q .
\end{gathered}
$$

Here $\langle\nu, S\rangle$ is defined as dual pairing of $S$ with the measure $\nu$, i.e.

$$
\langle\nu, S\rangle:=\int_{\mathbb{R}^{m \times n}} S(A) \mathrm{d} \nu(A) .
$$

Remark 2.2. If the Young-measure $\nu$ is a Dirac measure for almost every $(x, t) \in Q$, then $u$ is a weak solution of the elastodynamical system (2.1)-(2.4).

Remark 2.3. For $\phi \in \mathcal{E}_{0}, S \in \mathcal{F}_{0}$ and $A \in \mathbb{R}^{m \times n}$ there holds

$$
\begin{aligned}
\phi(A) & \leq \beta\left(\|A\|^{2}+1\right), \\
|S(A)| & \leq \beta(\|A\|+1) .
\end{aligned}
$$

Remark 2.4. The condition that $\phi^{q c}$ is convex is automatically satisfied for $n=1$ or $m=1$. The condition is needed to establish an a priori energy estimate for the solutions of the time discretized problem. If such bounds exist, the condition is not necessary in the proof of Theorem 2.1.

Remark 2.5. Alternative approximation schemes which can ensure existence in the case where $\phi^{q c}$ is nonconvex will be discussed Section 6 .

\section{Numerical schemes And CONSTRUCTIVE EXISTEnCE PROOF}

The proof of Theorem 2.1 is based on a discretization in time, energy estimates, and relaxation for each time-step [13,21]. The limit for infinitely small time steps then provides the asserted regularity of the solution. Without loss of generality let $g \equiv 0$ (as a shift of $u$ to $u-g$ would result in a problem with homogeneous boundary conditions).

\subsection{Time discretization}

Definition 3.1. Given $u_{0}, v_{0}, g \equiv 0$, and $h>0$ with an integer $N=T / h$, find $\left(u^{h, j}\right)_{h, j}$ such that $u^{h, 0}=u_{0}$, $u^{h,-1}=u_{0}-h v_{0}$, and $u^{h_{j}} \in H_{0}^{1}\left(\Omega ; \mathbb{R}^{m}\right)$ satisfies, for $j=1,2 \ldots, N$,

$$
\frac{u^{h, j}-2 u^{h, j-1}+u^{h, j-2}}{h^{2}}-\operatorname{div} S\left(D u^{h, j}\right)=0 .
$$


In the following we abbreviate

$$
\begin{gathered}
v^{h, j}:=\frac{u^{h, j}-u^{h, j-1}}{h} \text { and } \\
E_{j}:=E^{h, j}:=\int_{\Omega}\left(\phi^{q c}\left(D u^{h, j}\right)+\frac{1}{2}\left|v^{h, j}\right|^{2}\right) .
\end{gathered}
$$

Theorem 3.2 (Discrete Solution). The relaxed time-discretized system

$$
\int_{\Omega}\left(\left\langle\nu^{h, j}, S\right\rangle D \zeta+\frac{u^{h, j}-2 u^{h, j-1}+u^{h, j-2}}{h^{2}} \zeta\right) \mathrm{d} x=0
$$

with $j \in \mathbb{N}$ and $\zeta \in H_{0}^{1}\left(\Omega ; \mathbb{R}^{m}\right)$ admits a solution $\left(u^{h, j}, \nu^{h, j}\right)$ where $u^{h, j} \in H_{0}^{1}\left(\Omega ; \mathbb{R}^{m}\right)$ and $\nu^{h, j}$ is a Youngmeasure with $\left\langle\nu^{h, j}, I d\right\rangle=D u^{h, j}$.

Proof. The discrete problem (3.1) reads as a minimization problem for the energy functional

$$
\begin{aligned}
W_{j}(v) & :=W\left(v ; u^{h, j-1}, u^{h, j-2}\right) \\
& :=\int_{\Omega}\left(\phi(D v)+\frac{\left|v-2 u^{h, j-1}+u^{h, j-2}\right|^{2}}{2 h^{2}}\right) .
\end{aligned}
$$

Any minimizer $u$ of $W_{j}$ is a solution of the discretized differential equations. But, in general, $W_{j}$ is not weakly lower semicontinuous (w.l.s.c.) since $\phi$ is not assumed to be quasiconvex. Hence the existence of a minimizing Sobolev function $u$ cannot be expected. To describe more general solution concepts we consider $W_{j}^{q c}$, the quasiconvexification of the energy function $W_{j}$, i.e.

$$
W_{j}^{q c}(v):=\int_{\Omega}\left(\phi^{q c}(D v)+\frac{\left|v-2 u^{h, j-1}+u^{h, j-2}\right|^{2}}{2 h^{2}}\right) .
$$

Since $W_{j}^{q c}$ is w.l.s.c. there exists a minimizer $u^{h, j}$ with

$$
\inf _{j} W_{j}(v)=\inf _{v} W_{j}^{q c}(v)=W_{j}^{q c}\left(u^{h, j}\right)
$$

Then, one can prove the theorem by relaxation theory as in [13].

\subsection{Space discretization}

For each time-step, a Galerkin discretization is needed for the time-discretized system (3.4). A uniform partition with mesh-size $\mathrm{d} x=L / M$ in $M$ subintervals $I_{1}, I_{2}, \ldots, I_{M}$ of $\Omega=(0, L)$ defines the first-order finite element spaces

$$
\begin{aligned}
\mathcal{S}_{h} & :=\left\{v_{h} \in C(\bar{\Omega}) \mid \forall k=1,2, \ldots, M, v_{h} \text { affine on } I_{k}\right\}, \\
H_{h, g} & :=\left\{v_{h} \in \mathcal{S}_{h} \mid v_{h}=g \text { on } \partial \Omega\right\}, \quad \text { and } H_{h, 0}:=\mathcal{S}_{h} \cap H_{0}^{1}(\Omega) .
\end{aligned}
$$

On each interval $I_{k}:=((k-1) \mathrm{d} x, k \mathrm{~d} x)$, the Young-measure $\nu^{h, j}$ is discretized as a finite sum of $q$ Dirac measures. The chosen supports $F_{1}, \ldots, F_{q}$ discretize $\mathbb{R}$ and each discrete Young-measure assumes the representation

$$
\left.\nu^{h, j}\right|_{I_{k}}=\sum_{\ell=1}^{q} \lambda_{j, k, \ell} \delta_{F_{\ell}} \quad \text { on } I_{k}, k=1, \ldots, M
$$


with unknown convex coefficients

$$
\lambda_{j, k, 1}, \ldots, \lambda_{j, k, q} \geq 0 \quad \text { with } \sum_{\ell=1}^{q} \lambda_{j, k, \ell}=1
$$

and subject to the side-restriction

$$
\sum_{\ell=1}^{q} \lambda_{j, k, \ell} F_{\ell}=\partial u^{h, j} /\left.\partial x\right|_{I_{k}}
$$

Then define

$$
\begin{aligned}
Y M^{h}:= & \left\{\nu^{h, j} \text { Young-measure } \mid \nu^{h, j}\right. \text { satisfies (3.5) for coefficients } \\
& \text { with }(3.6)\} \\
\mathcal{B}^{j}:= & \left\{\left(u_{h}^{h, j}, \nu^{h, j}\right) \in H_{h, g} \times Y M^{h} \mid u^{h, j} \text { and } \nu^{h, j} \text { satisfies (3.7) }\right\}
\end{aligned}
$$

Definition 3.3 (Fully discrete Problem). Given $u_{0}, v_{0}, g, h>0, \mathrm{~d} x>0$ such that $N=T / h$ and $M=L / \mathrm{d} x$ are integers, set $u^{h, 0}=u_{0}, u^{h,-1}=u_{0}-h v_{0}$ at the nodes $x_{k}=k \mathrm{~d} x$ for $k=0,1, \ldots, M$ and for all $j=1, \ldots, N$, find $\left(u^{h, j}, \nu^{h, j}\right) \in \mathcal{B}^{j}$ which satisfies

$$
\int_{\Omega}\left(\left\langle\nu^{h, j}, S\right\rangle v_{h}+h^{-2}\left(u^{h, j}-2 u^{h, j-1}+u^{h, j-2}\right) v_{h}\right) \mathrm{d} x=0
$$

for all test functions $v_{h} \in H_{h, 0}$.

Theorem 3.4. The fully discrete problem has a unique solution $u^{h, j}$.

Proof. The proof is based on the stationary situation within each time-step essentially given in $[6,24]$. The uniqueness of the discrete displacement variables results from the uniform monotonicity of the low-order timedifference term.

Remark 3.5. The uniqueness of the discrete Young-measures is related to the 2-well problem which defines the convex hull uniquely. In the discrete situation, however, it may be that the minimizing Young measure is non-unique. For instance, let $F_{1}, F_{2}, F_{3}$ and $F_{4}$ denote the four distinct real arguments with $\phi\left(F_{n}\right)=0.1$ in the situation for $\phi$ of Figure 1. Then, even though the mean $\left.u_{h}^{\prime}\right|_{I_{j}}=\bar{\nu}^{h, j}=\lambda_{1} F_{1}+\cdots+\lambda_{4} F_{4}=0$ is unique, there are infinitely many convex coefficients $\lambda_{1}, \ldots, \lambda_{4} \geq 0$ with $\lambda_{1} F_{1}+\cdots+\lambda_{4} F_{4}=0$ and $\lambda_{1}+\cdots+\lambda_{4}=1$. Each of those solutions defines a different Young-measure which may be part of one different solution of the fully discrete problem.

Remark 3.6. The effective numerical solution is performed with a multilevel active-set strategy of [6] based on the Weierstrass maximum principle for Lagrange multipliers. The practical performance shows linear complexity in the number of unknowns [6].

Remark 3.7. Weak convergence as $\mathrm{d} x \rightarrow 0$ and $q \rightarrow \infty$ follows with the direct method of the calculus of variations, cf. [24] for details. Strong convergence is less obvious. We refer to [5] for a related problem (obtained for $q=\infty$ by convexification).

\subsection{Discrete energy estimate}

In order to prove the convergence of the discrete solution to a solution of the original problem it is necessary to obtain some a priori energy estimates for the discrete solutions which are independent of the discretization parameter $h>0$. We can prove the following result: 
Theorem 3.8 (Discrete Energy Estimate). Suppose $\phi^{q c}$ is convex, that $\alpha$ is as in Theorem 2.1, and that $u^{h, j}, v^{h, j}$ solve (3.1)-(3.3) for all $j=1, \ldots, N$. Then, for all $j=1, \ldots, N$,

$$
\left\|u^{h, j}\right\|_{H^{1}}^{2}+\left\|v^{h, j}\right\|^{2} \leq \frac{1}{\alpha}\left(2 E_{0}+|\Omega|\right) \quad \text { and } \quad E_{j} \leq E_{0}<\infty .
$$

Proof. Proposition 3.6 in [2] reads $D \phi^{q c}\left(\nabla u^{h, j}\right)=\left\langle\nu^{h, j}, S\right\rangle$. Multiplication of the discrete elasticity equation with $v^{h, j}$, integration in space and summing over all $j$ leads to

$$
\int_{\Omega} \sum_{j} \frac{v^{h, j}-v^{h, j-1}}{h} v^{h, j} \mathrm{~d} x=-\int_{\Omega} \sum_{j} D \phi^{q c}\left(\nabla u^{h, j}\right) \frac{\nabla v^{h, j}}{h} \mathrm{~d} x .
$$

Since $\phi^{q c}$ is convex by assumption one can use Jensen's inequality to obtain from this the discrete energy inequality (3.8).

\subsection{Weak convergence of the time discretization}

Theorem 3.9. The solutions $u^{h, j}, v^{h, j}$ of the time discretized problem are convergent as $h \rightarrow 0$ if and only if they satisfy the a priori estimate (3.8).

For the proof considers the piecewise constant and piecewise affine interpolations of the nodal values $\left(u^{h, j}\right)$, $\left(v^{h, j}\right),\left(v^{h, j}-v^{h, j-1}\right) / h$ and $\left(\nu^{h, j}\right)$. The separability of $\mathcal{F}_{0}$ and $\mathcal{E}_{0}$ is used to obtain a weakly converging subsequence for the Young-measures $\left(\nu^{h, j}\right)$. The discrete energy inequality yields bounds for the other interpolations. The technicalities follow arguments of [12] (in a slightly different notation) and hence are suppressed in the sequel.

For $h>0, j \in \mathbb{N}$, and the characteristic function $\chi^{h, j}:=\chi_{[h j, h(j+1)]}$ of the set $[h j, h(j+1)]$ for $j h<t<$ $h(j+1)$, where $\chi_{A}(x):=1$ if $x \in A$ and $\chi_{A}(x)=0$ elsewhere.

$$
\begin{aligned}
& w^{h}(t):=\sum_{j} \chi^{h, j}(t) \frac{v^{h, j+1}-v^{h, j}}{h} \\
& \text { (step function approximation of } u_{t t} \text { ), } \\
& \tilde{v}^{h}(t):=\sum_{j} \chi^{h, j}(t)\left(v^{h, j}+\frac{v^{h, j+1}-v^{h, j}}{h}(t-h j)\right) \\
& \text { (the primitive of } w^{h} \text { ), } \\
& v^{h}(t):=\sum_{j} \chi^{h, j}(t) v^{h, j+1} \quad\left(\text { step function approximation of } u_{t}\right), \\
& \tilde{u}^{h}(t):=\sum_{j} \chi^{h, j}(t)\left(u^{h, j}+v^{h, j+1}(t-h j)\right) \quad(\text { its primitive) } \\
& u^{h}(t):=\sum_{j} \chi^{h, j}(t) u^{h, j+1} \quad(\text { step function approximation of } u) \\
& \nu^{h}:=\left(\nu_{x, t}^{h}\right)_{(x, t)}:=\sum_{j} \chi^{h, j}(t) \nu_{x}^{h, j+1} \quad \text { (Young-measure). }
\end{aligned}
$$

One can prove that the Young-measure $\nu^{h}$ is generated by the sequence $\left(\sum_{j} \chi^{h, j}(t) D u^{h, j, k}\right)_{k}$. Moreover there holds

$$
\nu^{h} \in L_{l o c}^{1}\left(\Omega \times\left(0, T_{0}\right) ; \mathcal{E}_{0}^{\prime}\right) \cap L_{l o c}^{2}\left(\Omega \times\left(0, T_{0}\right), \mathcal{F}_{0}^{\prime}\right)
$$


With $\nu^{h}$ and $w^{h}$ one can recast (3.1) for $t \geq h$ and $\zeta \in H_{0}^{1}\left(\Omega ; \mathbb{R}^{m}\right)$ into

$$
\int_{\Omega}\left(\left\langle\nu_{x, t}^{h}, S\right\rangle D \zeta+w^{h}(x, t) \zeta\right) \mathrm{d} x=0 .
$$

An integration over $\left(h, T_{0}\right)$ leads to

$$
\int_{h}^{T_{0}} \int_{\Omega}\left(\left\langle\nu_{x, t}^{h}, S\right\rangle D \zeta-\tilde{v}^{h}(x, t) \zeta_{t}(x)\right) \mathrm{d} x \mathrm{~d} t=0, \quad \text { for all } \zeta \in H_{0}^{1}
$$

Then one proves $D u^{h}=\left\langle\nu^{h}, I d\right\rangle$. A short calculation shows

$$
\tilde{u}^{h}(\cdot, 0)=u_{0}, \quad \text { and } \quad \tilde{v}_{t}^{h}(\cdot, 0)=v_{0} .
$$

The aim is to prove that $u^{h}$ and $\nu^{h}$ converge in an appropriate norm to a solution of the original problem. One observes that $\left(\nu^{h}\right)_{h}$ is bounded in $L_{\text {loc }}^{2}\left(\Omega \times \mathbb{R}^{+} ; \mathcal{F}_{0}^{\prime}\right)$. Since $\mathcal{F}_{0}$ is separable,

$$
L_{\text {loc }}^{2}\left(\Omega \times \mathbb{R}^{+} ; \mathcal{F}_{0}^{\prime}\right) \cong\left(L_{\text {loc }}^{2}\left(\Omega \times \mathbb{R}^{+} ; \mathcal{F}_{0}\right)\right)^{\prime} .
$$

Hence the bounded sequence $\left(\nu^{h}\right)_{h}$ has a subsequence (not relabeled) which converges weakly in $\left(L_{\text {loc }}^{2}\left(\Omega \times \mathbb{R}^{+} ; \mathcal{F}_{0}\right)\right)^{\prime}$. Therefore for all $g \in L_{l o c}^{2}\left(\Omega \times \mathbb{R}^{+} ; \mathcal{F}_{0}\right)$,

$$
\left(\int_{0}^{T_{0}} \int_{\Omega}\left\langle\nu_{x, t}^{h}, g_{x, t}\right\rangle \mathrm{d} x \mathrm{~d} t\right)_{h} \rightarrow \int_{0}^{T_{0}} \int_{\Omega}\left\langle\nu_{x, t}, g_{x, t}\right\rangle \mathrm{d} x \mathrm{~d} t .
$$

For test functions $g_{x, t}(y)=f(y) \zeta(x, t)$ with $\zeta \in L_{l o c}^{2}\left(\Omega \times \mathbb{R}^{+}\right)$and $f \in \mathcal{F}_{0}$, this yields

$$
\left(\left\langle\nu^{h}, f\right\rangle\right)_{h} \rightarrow\langle\nu, f\rangle \quad \text { in } L_{l o c}^{2}\left(\Omega \times \mathbb{R}^{+}\right) .
$$

The same arguments show

$$
\left(\nu^{h}\right)_{h} \rightarrow \nu \quad \text { in } L_{l o c}^{2}\left(\Omega \times \mathbb{R}^{+} ; \mathcal{F}_{0}^{\prime}\right) \cap L_{l o c}^{1}\left(\Omega \times \mathbb{R}^{+} ; \mathcal{E}_{0}^{\prime}\right) .
$$

Moreover, there exists a diagonal sequence of $\left(\sum_{j} \chi^{h, j}(t) u^{h, j, k}\right)_{h, k}$ which generates the Young-measure $\nu$. Since $D \phi, D \phi^{q c} \in \mathcal{F}_{0}$ leads to

$$
\begin{aligned}
\left(\left\langle\nu^{h}, D \phi\right\rangle\right)_{h} & \rightarrow\langle\nu, D \phi\rangle, \\
\left(\left\langle\nu^{h}, D \phi^{q c}\right\rangle\right)_{h} & \rightarrow\left\langle\nu, D \phi^{q c}\right\rangle \quad \text { in } L_{l o c}^{2}\left(\Omega \times \mathbb{R}^{+}\right) .
\end{aligned}
$$

Now the discrete energy inequality allows to prove the following a priori bounds:

$$
\sup _{0 \leq t \leq T_{0}}\left(\left\|u^{h}(t)\right\|_{H_{0}^{1}}+\left\|\tilde{u}^{h}(t)\right\|+\left\|v^{h}(t)\right\|+\left\|v^{h}(t)\right\|+\left\|\tilde{v}^{h}(t)\right\|_{H^{-1}}+\left\|w^{h}(t)\right\|_{H^{-1}}+\left\|\tilde{v}^{h}(t)\right\|\right) \leq C .
$$

Then the weak- $\star$ compactness property yields subsequences with

$$
\begin{aligned}
& \left(u^{h}\right) \stackrel{\star}{\rightarrow} u \quad \text { in } L^{\infty}\left(\left(0, T_{0}\right) ; H_{0}^{1}\right), \\
& \left(\tilde{u}^{h}\right) \stackrel{\star}{*} \quad \text { in } W^{1, \infty}\left(\left(0, T_{0}\right) ; L^{2}\right), \\
& \left(v^{h}\right) \stackrel{\star}{*} v \quad \text { in } L^{\infty}\left(\left(0, T_{0}\right) ; L^{2}\right), \\
& \left(\tilde{v}^{h}\right) \stackrel{\star}{*} \quad \text { in } W^{1, \infty}\left(\left(0, T_{0}\right) ; H^{-1}\right) \cap L^{\infty}\left(\left(0, T_{0}\right) ; L^{2}\right) .
\end{aligned}
$$


With Lemma 6.3 from [15] one proves that $\tilde{u}=u, \tilde{v}=v$.

In the limit $h \rightarrow 0$, the weak convergence of $\left(\nu^{h}\right)_{h}$ leads to

$$
\operatorname{supp} \nu \subset\left\{a \mid \phi(a)=\phi^{q c}(a)\right\} \quad \text { almost everywhere. }
$$

Moreover we get

$$
\int_{0}^{T_{0}} \int_{\Omega}\left(\langle\nu, S\rangle D \zeta-u_{t} \zeta_{t}\right) \mathrm{d} x \mathrm{~d} t=0 \quad \text { for all } \zeta \in H_{0}^{1}\left(\Omega \times\left(0, T_{0}\right)\right) .
$$

Furthermore, by the weak convergence of $\left(\nu^{h}\right)_{h},\left\langle\nu^{h}, I d\right\rangle \rightarrow\langle\nu, I d\rangle$. But on the other hand, $\left\langle\nu^{h}, I d\right\rangle=D u^{h} \rightarrow$ $D u$. Hence $D u=\langle\nu, I d\rangle$ which concludes the proof.

\subsection{Extensions to weaker differentiability conditions}

We can slightly enlarge the class of energy functionals for which Theorem 2.1 holds. In particular we want to allow functions with cusps. A typical example is the 2 -well energy density $\phi(Y):=\operatorname{dist}(Y,\{-1,+1\})^{2}$ plotted in Figure 1.

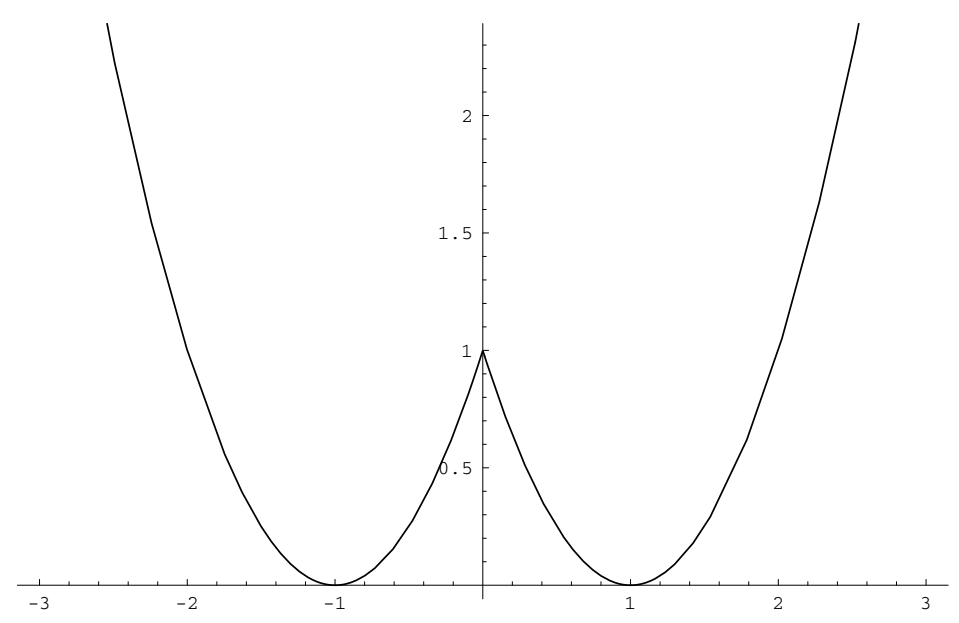

Figure 1. Plot of the energy function $\phi(Y):=\operatorname{dist}(Y,\{-1,+1\})^{2}$ for $-3 \leq Y \leq+3$. The cusp of $\phi$ in $Y=0$ does not lead to a lack of differentiability for $\phi^{q c}$.

Theorem 3.10 (Weakening of the differentiability condition). If $\phi$ is not a $\mathcal{C}^{1}$-function, but if, for all $B \in$ $\mathbb{R}^{m \times n}$, there exists a linear mapping $L$ from $\mathbb{R}^{m \times n}$ to $\mathbb{R}^{m \times n}$, such that

$$
\phi(A) \leq \phi(B)+L(A-B)+o\left(|A-B|^{2}\right)
$$

for all $A \in \mathbb{R}^{m \times n}$, then there exists a Young-measure solution of (2.1)-(2.3) in the sense of Theorem 2.1.

Proof. The condition (3.15) is sufficient to use the results from [2] (in particular Prop. 3.6). The proof of Theorem 2.1 considers the full derivatives of $\phi$ solely after testing by the corresponding Young-measures. Their support is contained in the set where $\phi=\phi^{q c}$ and is $\mathcal{C}^{1}$. Hence the above arguments prove Theorem 3.10 as well. 


\section{Numerical Realization}

This section is devoted to the multilevel adaptation of [6] for the scientific computation of one-dimensional nonconvex elastodynamics based on the algorithm of Section 3. For the time-step width $h$ and given initial values $u_{0}, u_{1}$, set $u^{h, 0}=u_{0}, \quad u^{h,-1}=u_{0}-h v_{0}$, and let $u^{h, j}$ solve

$$
\frac{u^{h, j}-2 u^{h, j-1}+u^{h, j-2}}{h^{2}}-\operatorname{div} S\left(D u^{h, j}\right)=0
$$

In each time step we have to minimize the energy functional

$$
\begin{aligned}
W^{h, j}(v) & :=W_{h}\left(v ; u^{h, j-1}, u^{h, j-2}\right) \\
& :=\int_{\Omega} \phi(D v)+\frac{\left|v-2 u^{h, j-1}+u^{h, j-2}\right|^{2}}{2 h^{2}}
\end{aligned}
$$

subject to the boundary conditions $v \in H_{0}^{1}(\Omega)$. The minimization problem (4.2) is nonconvex and, in general, admits no classical solution. Instead, we can find a pair $\left(u^{h, j}, \nu^{h, j}\right)$, where $u^{h, j} \in H_{0}^{1}(\Omega), \nu^{h, j}=\left(\nu_{x}^{h, j}\right)_{x}$ is a probability measure, and $\left\langle\nu^{h, j}, I d\right\rangle=D u^{h, j}$ for almost every $x \in \Omega$, such that $\left(u^{h, j}, \nu^{h, j}\right)$ minimizes

$$
\widetilde{W}^{h, j}(v, \mu):=\int_{\Omega}\langle\phi, \mu\rangle+\frac{\left|v-2 u^{h, j-1}+u^{h, j-2}\right|^{2}}{2 h^{2}} .
$$

The one-dimensional domain $\Omega=(0,1)$ is split into a finite number of intervals of length $\ell=\mathrm{d} x$. Then let $U_{\ell}$ denotes the space of all continuous functions which are affine on each discretization interval of $\Omega$ and satisfy the boundary conditions (e.g. Dirichlet boundary conditions on $\partial \Omega$ ).

On each element, the Young-measure $\nu$ is approximated by a finite sum of Dirac measures $\delta_{F_{i}}$ with unknown weights $\lambda_{j}, 0 \leq \lambda_{j} \leq 1$ and $\sum_{j} \lambda_{j}=1$, and prescribed atoms $F_{j}$. Details on the static problem are given in [6].

The support of the approximating measure given by $\left(F_{j}\right)_{j=1, \ldots, K}$ is chosen as $F_{j}:=-A+2 A j / K$ with $A=2$ and $K=12$. The precise value of $A$ is not crucial if it is only chosen sufficiently larger than a critical value given by $\sup \left|u_{x}\right|$.

In the time evolution problem, at hand, we have only weak convergence in the limit for $h \rightarrow 0$ (where $h>0$ is the time-step width); we do not expect a priori error estimates.

By the approximation of the Young-measure described above, the time step problem reduces to the minimization of the functional

$$
\widetilde{W}_{\text {approx }}^{h, j}=\int_{\Omega}\left(\sum_{k} \lambda_{k}(x) \phi\left(F_{k}\right)+\frac{1}{2 h}\left|v(x)-2 u^{h, j}(x)+u^{h, j-1}(x)\right|^{2}\right) \mathrm{d} x
$$

over all $\lambda_{k} \geq 0$ and $v \in U_{\ell}$ with $\sum_{k} \lambda_{k}(x) F_{k}=v(x)$ and $\sum_{k} \lambda_{k}(x)=1$.

Problem (4.4) is a discrete optimization problem. In fact it is (independent of the choice of $\phi$ ) a quadratic problem and hence solvable by standard optimization software (qp in Matlab).

A flow chart on the algorithm is depicted in Figure 2. For a description of how to select active grid points for the approximative measure and how to check the need for activating more grid points we refer to [6]. The complete program was firstly tested on the problem of the linear wave equation, where the true solutions are well-known. The approximated solution shows an error for large times, a possible consequence of numerical viscosity. The numerical viscosity can be reduced by choosing a fine discretization of the Young-measure (i.e. $K$ large) and this has been observed experimentally.

As in linear elasticity we have to choose $h$ sufficiently small with respect to $\ell$. In our experiments we follow the Courant-Friedrichs-Lewy (CFL) stability condition $h / \ell \leq\left|\phi_{x x}\right|$. 


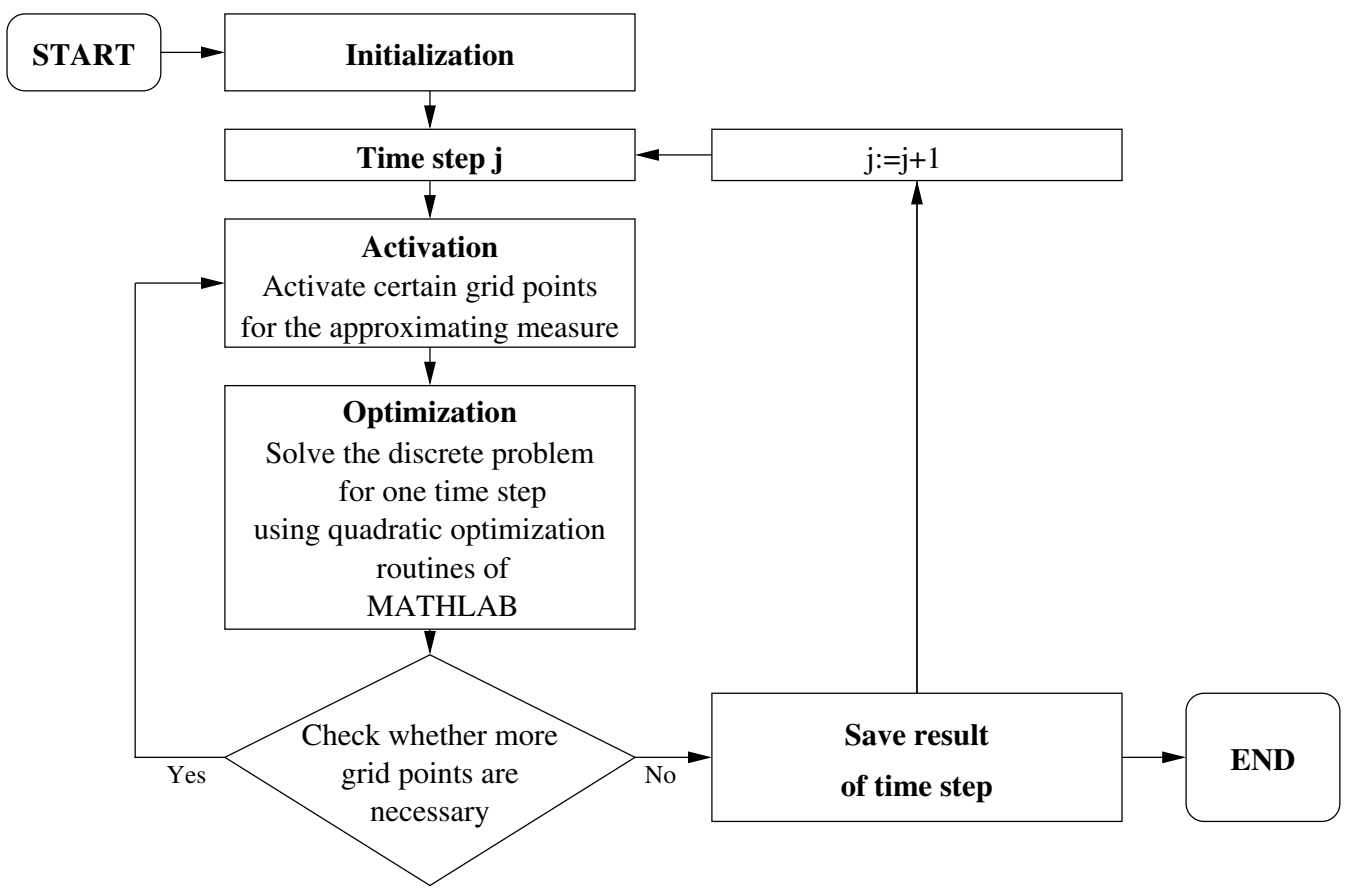

FiguRE 2. Overview on the implemented algorithm.

TABLE 1. Overview of presented numerical experiments. (SMA abbreviates shape memory alloy.)

\begin{tabular}{|ll|lcc|}
\hline Sec. & Label & Description & $\mathrm{d} x$ & $h$ \\
\hline 5.1 & Exp. A & 2-well potential, initially two peaks & 0.02 & 0.02 \\
\hline 5.2 & Exp. B & Sinus on the boundary, stopping & 0.05 & 0.05 \\
& Exp. C & Small sinus on the boundary, stopping & 0.05 & 0.05 \\
& Exp. D & Sinus on the boundary, decaying & 0.05 & 0.05 \\
& Exp. E & Sinus on the boundary, long time & 0.05 & 0.05 \\
\hline 5.3 & Exp. F & SMA with decreasing temperature & 0.02 & 0.02 \\
& Exp. G & SMA with increasing temperature & 0.02 & 0.02 \\
\hline
\end{tabular}

\section{Numerical EXAMPLES}

In this section we present several one-dimensional numerical simulations of elastodynamics with a material that allows phase transitions. The seven simulations $A, B, \ldots, G$ summarized in Table 1 fall into three different categories with emphasis on initial conditions (Sect. 5.1), time-depending boundary conditions (Sect. 5.2), and changing temperature (Sect. 5.3). The variable $u(x, t)$ physically describes either the elongation or transversal displacement at $x \in \Omega$ of the beam $\Omega=(0,1)$ at time $t$. Microscopic oscillations of the one-dimensional deformation gradient are described by the Young measure $\nu_{x, t}$. The algorithm of Section 4 produced numerical approximations $u^{h}(x, t)$ and $\nu_{x, t}^{h}$ indicated through their expectation values $u_{x}^{h}(x, t)$. We emphasize that the microstructure is encoded in the measure $\nu_{x, t}^{h}$ and is infinitely fine, hence it is not immediately visible in the following pictures that describe only the macroscopic deformation.

The 2 -well potential $\phi(F):=\min \left\{|F-1|^{2},|F+1|^{2}\right\}$ of Figure 1 models a two-phase shape memory alloy (SMA) in the one-dimensional body $\Omega$ with $g=0$ and $v_{0}=0$. 


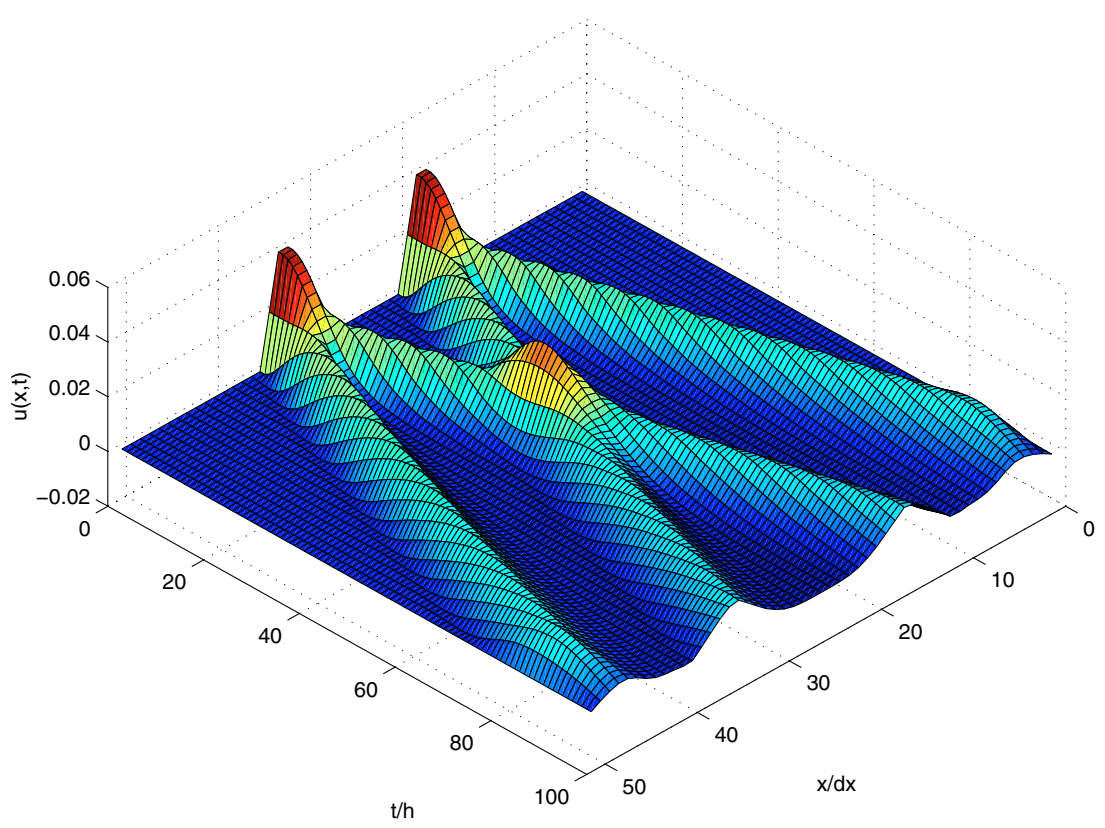

FiguRE 3. Numerical solution $u^{h, j}$ in Experiment A for $\mathrm{d} x=0.02$ and $h=0.02$ plotted over the space-time domain $(0,1) \times(0,1.96)$ in units of $t / h$ and $x / \mathrm{d} x$.

\subsection{Homogenous Dirichlet boundary conditions}

At fixed temperature, a clamped beam of a shape memory alloy is elongated by an initial displacement $u_{0}$.

$$
u_{0}(x)=\max \{0, .05-|x-.35|, .05-|x-.65|\} \quad \text { and } \quad v_{0}(x)=0
$$

Experiment A. The algorithm of Figure 2 computed an approximative solution $u^{h, j}$ shown in Figure 3 . The discrete displacement for all $x / \mathrm{d} x=0,1, \ldots, 50$ and $t / h=0,1, \ldots, 100$ were stored in a $51 \times 101$ matrix plotted with Matlab. The different colors correspond to the height given by $u^{h, j}$. The macroscopic strain $u_{x}(x, t)$ is simulated by the average of the Young-measure approximation

$$
\bar{\nu}_{h, j}=\left\langle\nu^{h, j}, \mathrm{Id}\right\rangle=u_{x}^{h, j}
$$

and plotted in Figure 4.

The approximation of $u_{x}$ (i.e. the expectation value of $\nu^{h, j}$ or the averaged microstructure) is given with different colors indicated in the color bar for values between -1 (blue) and 1 (red).

We observe that traveling waves intersecting with each other start from the elongated points of the initial state. A numerical comparison with the linear wave equation (not displayed) shows that the form of the traveling waves is similar to, but more triangular like than those in the linear case.

\subsection{Phase transformation induced through the boundary}

In a minor generalization of (2.1)-(2.4) of the last experiment we choose the Dirichlet boundary condition $u=g(x, t)$ on $\partial \Omega$ time dependent. This simulates different prescribed oscillations of one end of the wire. With $u_{0} \equiv 0 \equiv v_{0}, u \equiv 0$ is a solution to the partial differential equation for homogeneous boundary conditions and $\nu=\frac{1}{2} \delta_{-1}+\frac{1}{2} \delta_{+1}$ is the corresponding Young-measure. 


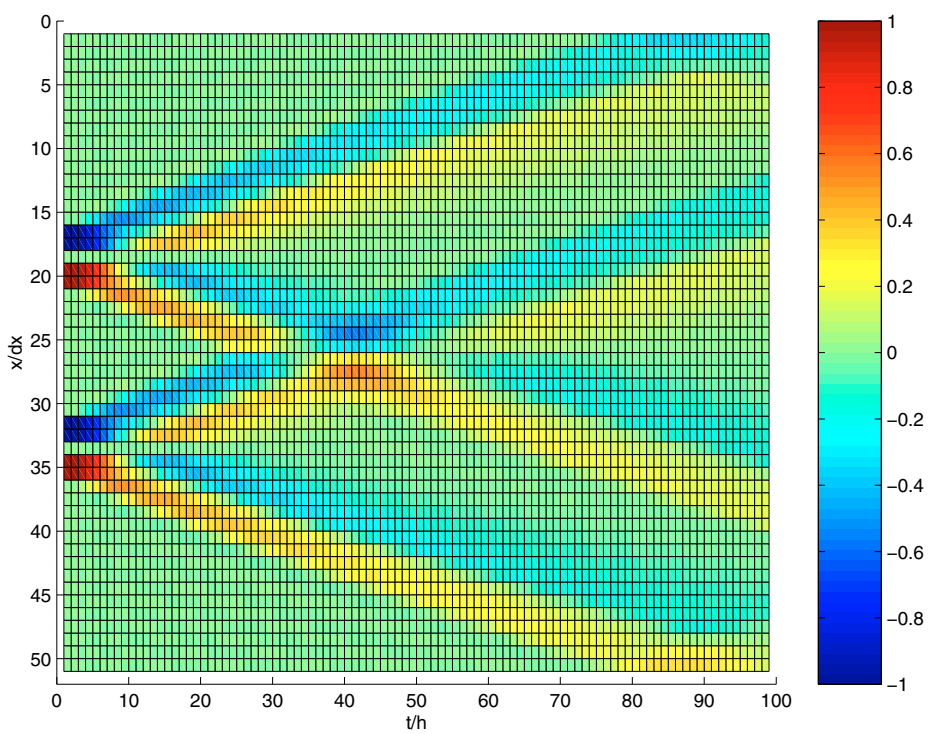

FiguRE 4. Macroscopic strain (5.1) (plotted in colors in the range between the wells -1 and +1 ) computed from Young-measure approximation $\nu^{h, j}$ in Experiment A plotted over $(0,1) \times$ $(0,1.96)$ in units of $t / h$ and $x / \mathrm{d} x$.

Experiment B. The time-dependent Dirichlet boundary condition reads

$$
u(1, t)=\left\{\begin{array}{cc}
\sin (2 \pi t) & \text { for } t \leq 2, \\
0 & \text { for } t>2,
\end{array} \text { and } \quad u(0, t)=0\right.
$$

while $u_{0} \equiv 0 \equiv v_{0}$. The computed solution $u^{h, j}$ to this problem is shown in Figure 5 . It is interesting to have a look at the gradient of $u$ (see Fig. 6): we observe that $u_{x}(x, t)$ is after some small time nearly everywhere close to one of the wells. This means that by applying a (fast) oscillation to the boundary of the wire we induce a phase separation in the material.

In the next experiments we investigate whether this effect does also hold for smaller amplitudes of the oscillation and for a smooth decay of the oscillations.

Experiment C. A modification of boundary conditions in Experiment B (by multiplying by 1/2), namely $u_{0} \equiv 0 \equiv v_{0}$ and

$$
u(1, t)=\left\{\begin{array}{cc}
\frac{1}{2} \sin (2 \pi t) & \text { for } t \leq 2, \\
0 & \text { for } t>2,
\end{array} \text { and } \quad u(0, t)=0 .\right.
$$

The result (see Fig. 7) is quite similar to the last experiment. In fact the phase separation seems not to dependent on the intensity of the boundary oscillations. This can be seen from Figure 8 where the distribution of different values for the gradients is shown. We observe clearly that the values of the gradient concentrate close to the wells -1 and +1 . The only difference to the last experiment is that the pattern of the different phases at a given time is finer as before. We expect that in the limit of vanishing oscillations this again approaches an infinitely fine mixture of phases.

Experiment D. Let the oscillations on the boundary decay slowly in time,

$$
u(1, t)=\left\{\begin{array}{cl}
\frac{3^{-t}}{2} \sin (2 \pi t) & \text { if } t \leq 2, \\
0 & \text { if } t>2,
\end{array} \quad \text { and } \quad u(0, t)=0 .\right.
$$

We observe a similar, but slightly more complex solution as in the examples before; see Figures 9 and 10 . 


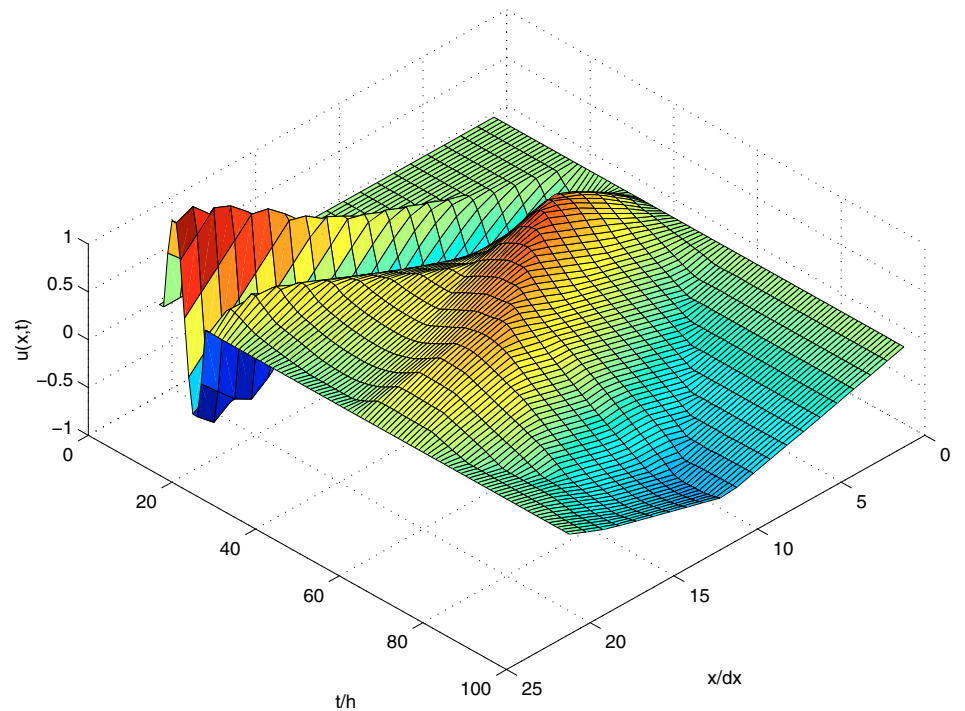

Figure 5. Numerical solution $u^{h, j}$ in Experiment B for $\mathrm{d} x=0.05$ and $h=0.05$ plotted over the space-time domain $(0,1) \times(0,4.9)$ in units of $t / h$ and $x / \mathrm{d} x$.

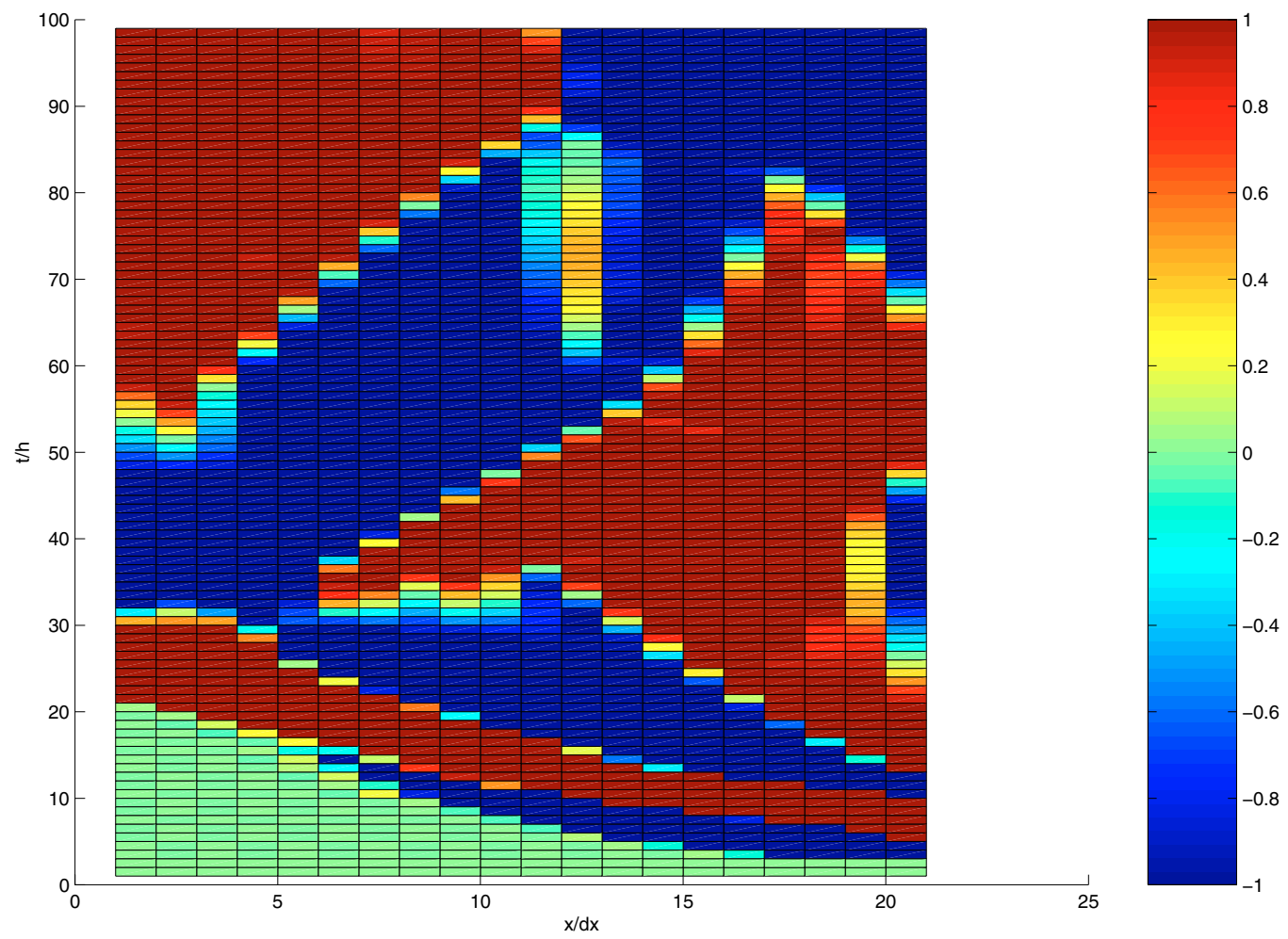

FiguRE 6. Macroscopic strain (5.1) (plotted in colors in the range between the wells -1 and +1 ) computed from Young-measure approximation $\nu^{h, j}$ in Experiment B plotted over $(0,1) \times(0,4.9)$ in units of $t / h$ and $x / \mathrm{d} x$. 


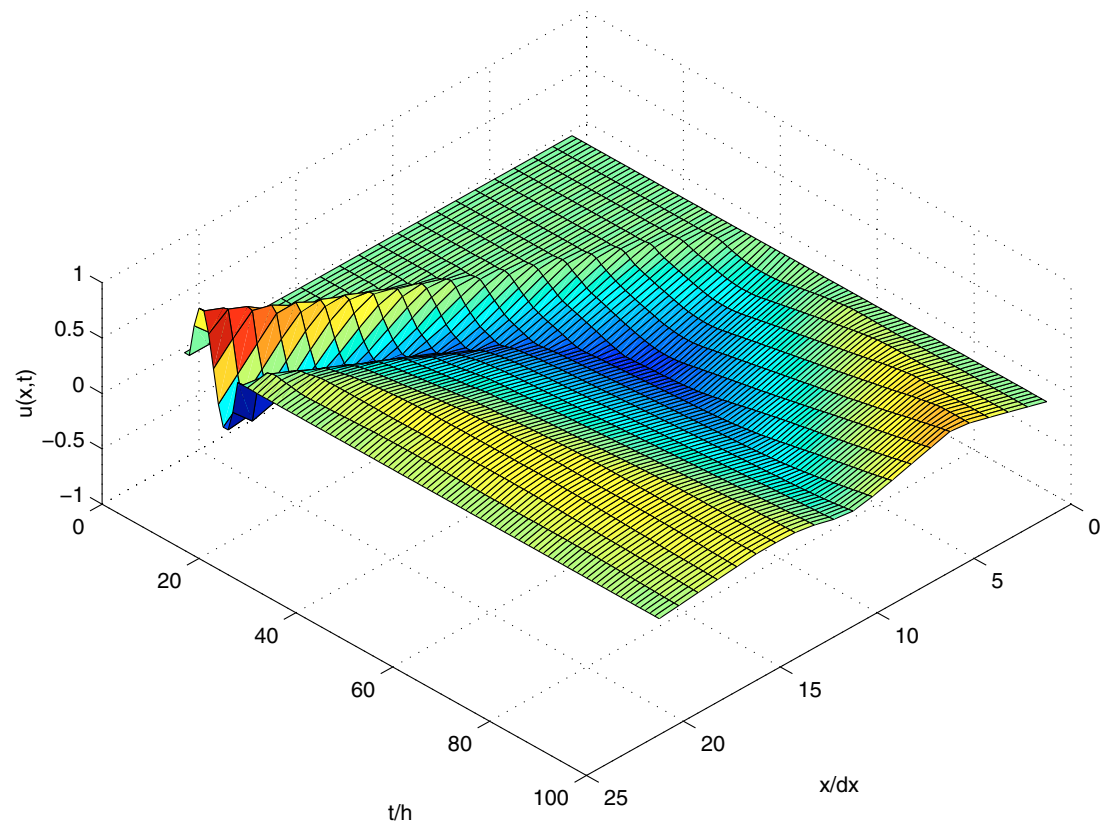

FiguRE 7. Numerical solution $u^{h, j}$ in Experiment $\mathrm{C}$ for $\mathrm{d} x=0.05$ and $h=0.05$ plotted over the space-time domain $(0,1) \times(0,4.9)$ in units of $t / h$ and $x / \mathrm{d} x$.

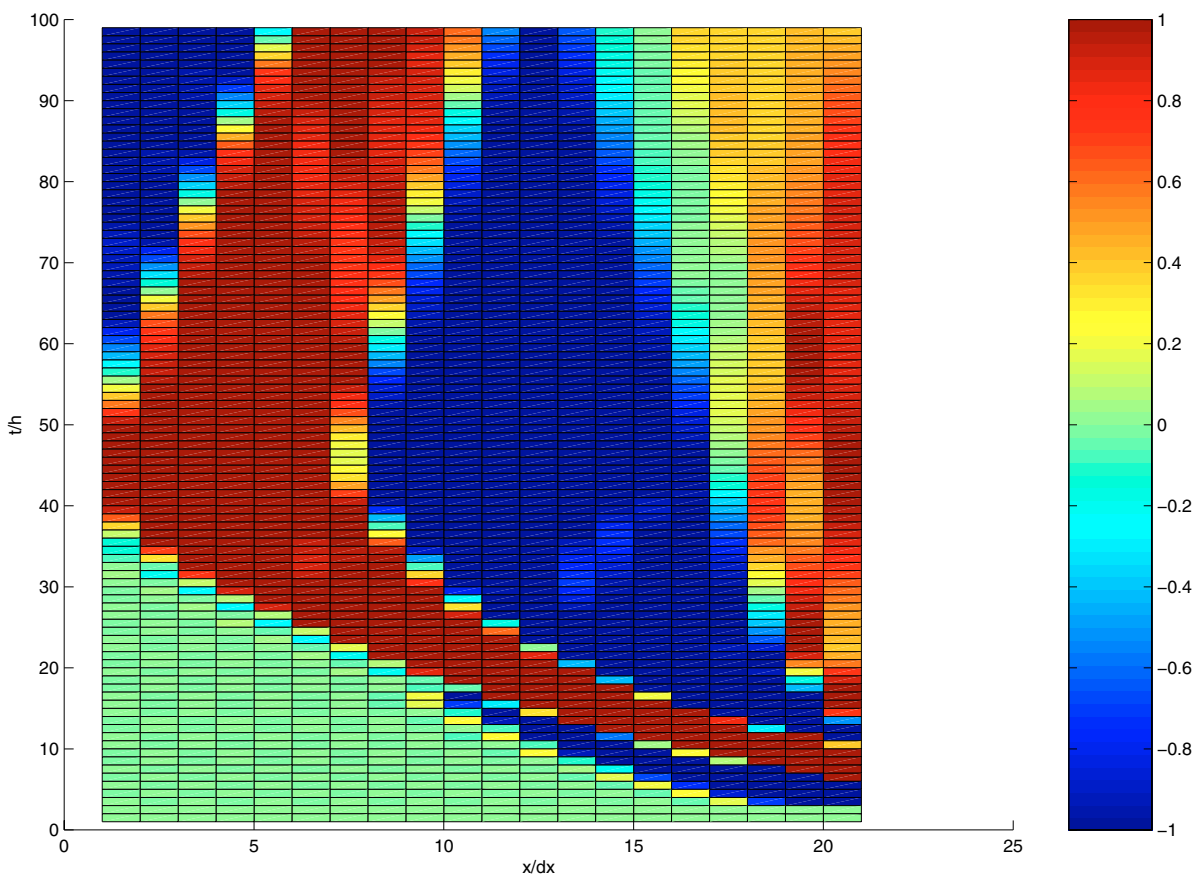

FiguRE 8. Macroscopic strain (5.1) (plotted in colors in the range between the wells -1 and +1 ) computed from Young-measure approximation $\nu^{h, j}$ in Experiment C plotted over $(0,1) \times(0,4.9)$ in units of $t / h$ and $x / \mathrm{d} x$. 


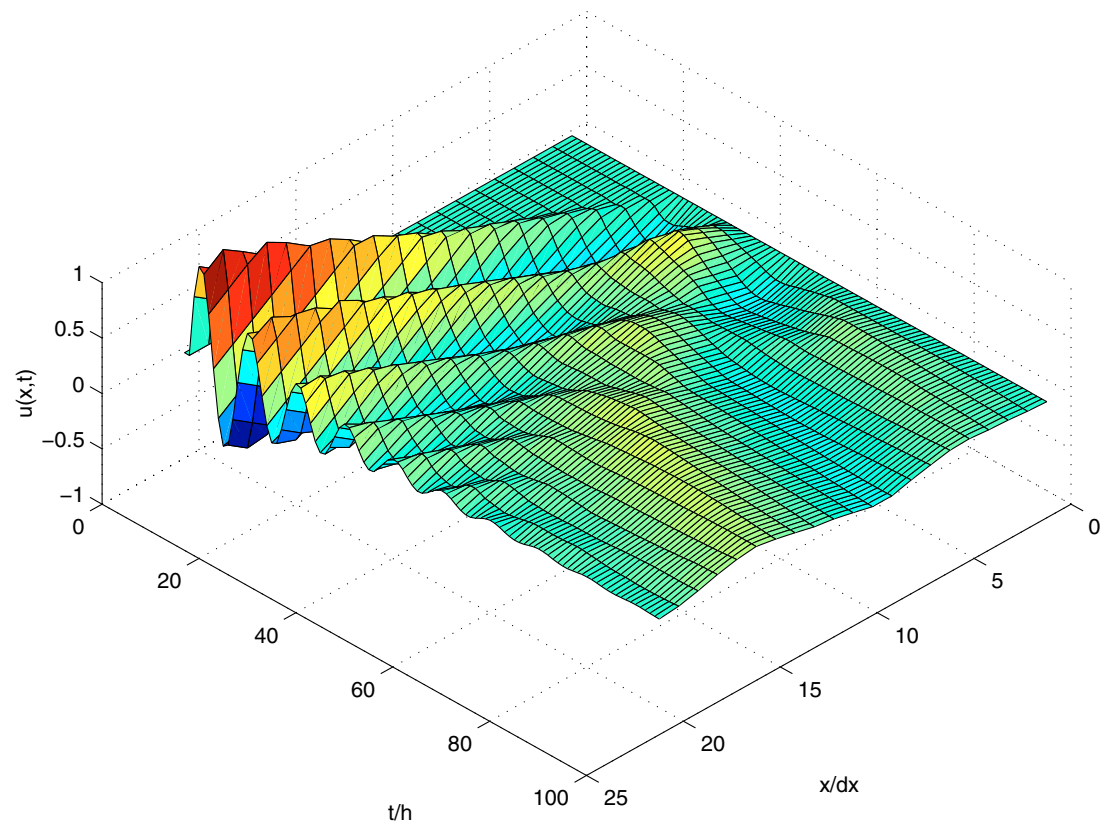

FiguRE 9. Numerical solution $u^{h, j}$ in Experiment D for $\mathrm{d} x=0.05$ and $h=0.05$ plotted over the space-time domain $(0,1) \times(0,4.9)$ in units of $t / h$ and $x / \mathrm{d} x$.

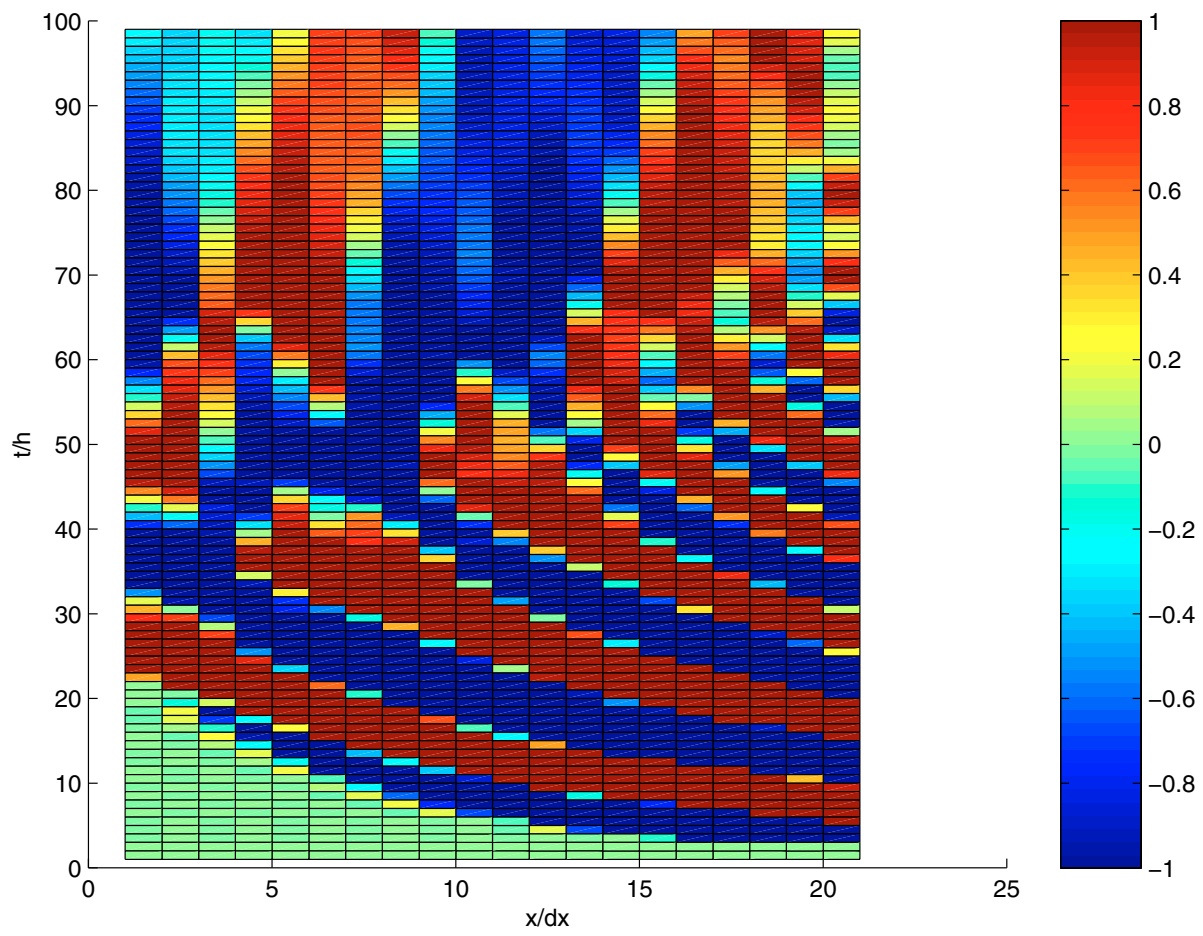

Figure 10. Macroscopic strain (5.1) (plotted in colors in the range between the wells -1 and +1 ) computed from Young-measure approximation $\nu^{h, j}$ in Experiment D plotted over $(0,1) \times(0,4.9)$ in units of $t / h$ and $x / \mathrm{d} x$. 


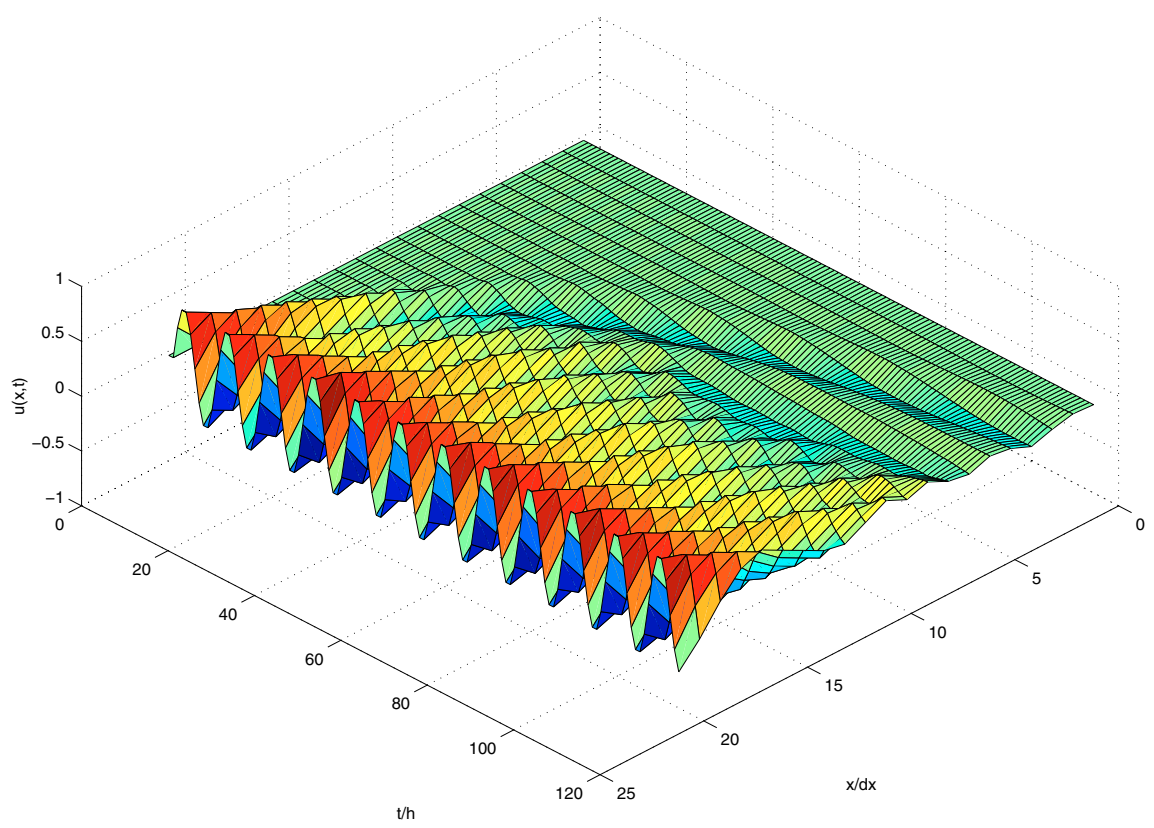

Figure 11. Numerical solution $u^{h, j}$ in Experiment $\mathrm{E}$ for $\mathrm{d} x=0.05$ and $h=0.05$ plotted over the space-time domain $(0,1) \times(0,5.9)$ in units of $t / h$ and $x / \mathrm{d} x$.

Experiment E. The experiments B-D had the slight drawback of showing the solution only for rather small times. To conclude the experiments with oscillating boundary conditions we show an example where the oscillating boundary condition are present over a longer time. The resulting displacement and the deformation (see Figs. 11 and 12) soon seem to approach a periodic solution, similar to the long time calculations above.

\subsection{Phase transition induced by temperature changes}

The third class of simulations concerns a time-dependent potential $\phi$. This models a shape memory alloy where the temperature is changed during the experiment and passes a critical value, at which the type of $\phi$ switches from nonconvex to convex or vice versa.

Experiment F. In this experiment we decrease the temperature below the critical temperature, thus inducing a phase transition from austenitic to martensitic phase. We choose the temperature-dependent potential

$$
\phi(F, \theta):=\theta+\min \left\{\frac{1}{2} F^{2}-\theta, \frac{1}{2} \min \left\{(F-1)^{2},(F+1)^{2}\right\}\right\},
$$

where the temperature $\theta(t)$ is chosen as $\theta(t):=0.2-t$, hence the potential is changing from a 3 -well to a 2-well potential. As initial condition we choose

$$
u_{0}(x):=0.1 \sin (2 \pi x) \quad \text { and } \quad v_{0}=0 .
$$

We observe that the initial oscillations essentially continue, but with decreasing amplitude (compare Fig. 13). This seems to be an effect of the change in the potential that takes energy out of the system. In view of the results of Experiment G (see below), numerical viscosity as a source of the decay in the amplitude can be excluded. 


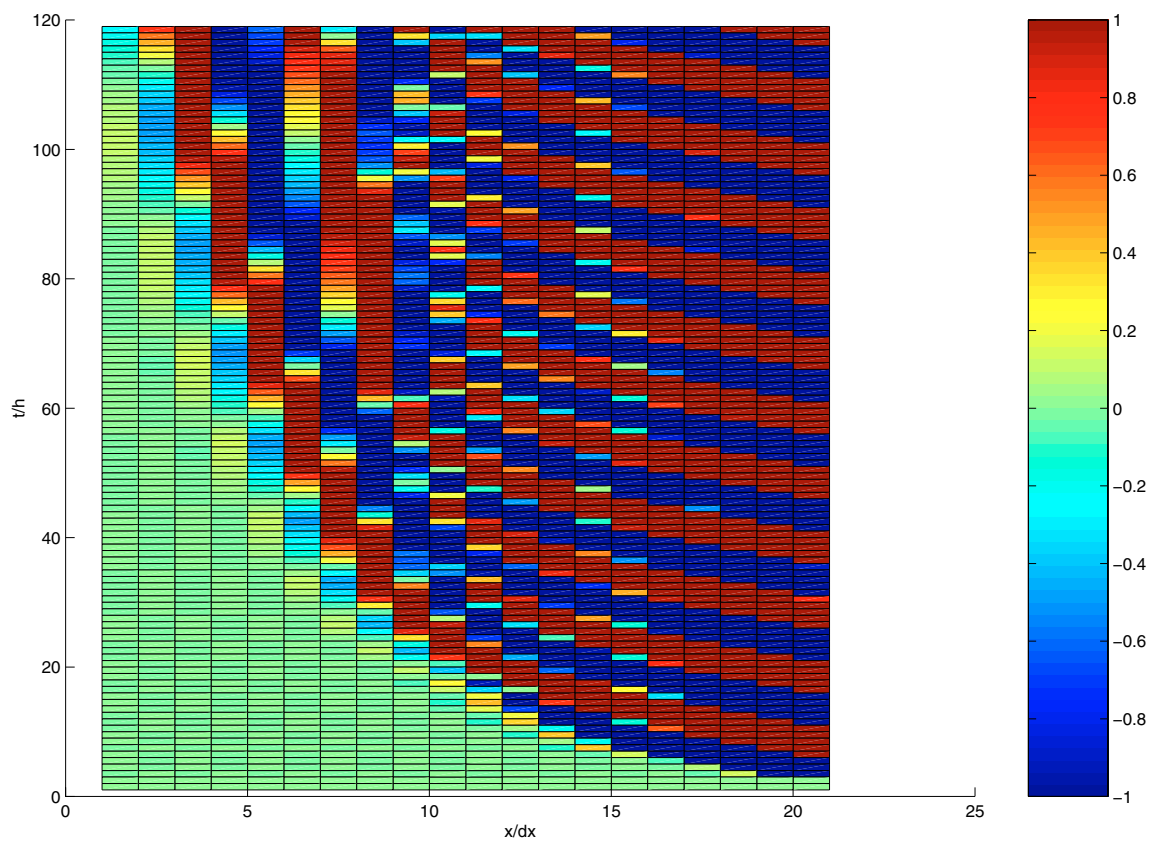

Figure 12. Macroscopic strain (5.1) (plotted in colors in the range between the wells -1 and +1 ) computed from Young-measure approximation $\nu^{h, j}$ in Experiment E plotted over $(0,1) \times(0,5.9)$ in units of $t / h$ and $x / \mathrm{d} x$.

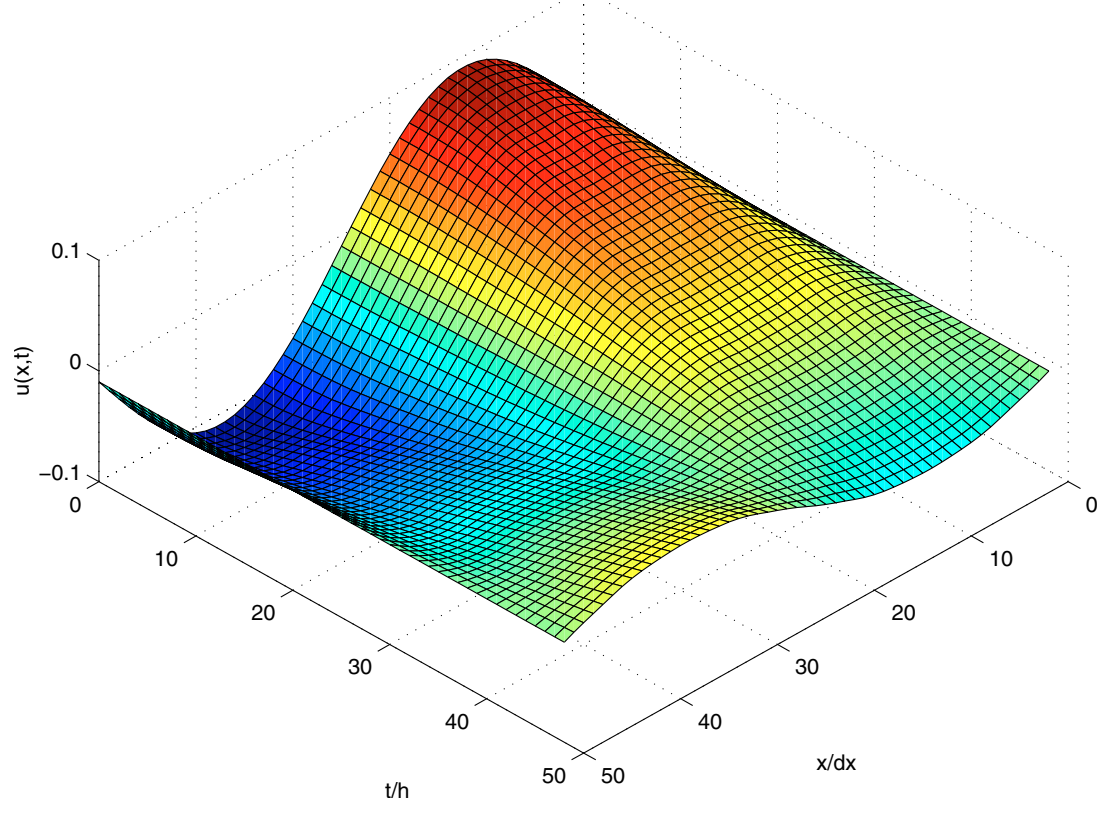

Figure 13. Numerical solution $u^{h, j}$ in Experiment $\mathrm{F}$ for $\mathrm{d} x=0.02$ and $h=0.02$ plotted over the space-time domain $(0,1) \times(0,1)$ in units of $t / h$ and $x / \mathrm{d} x$. 


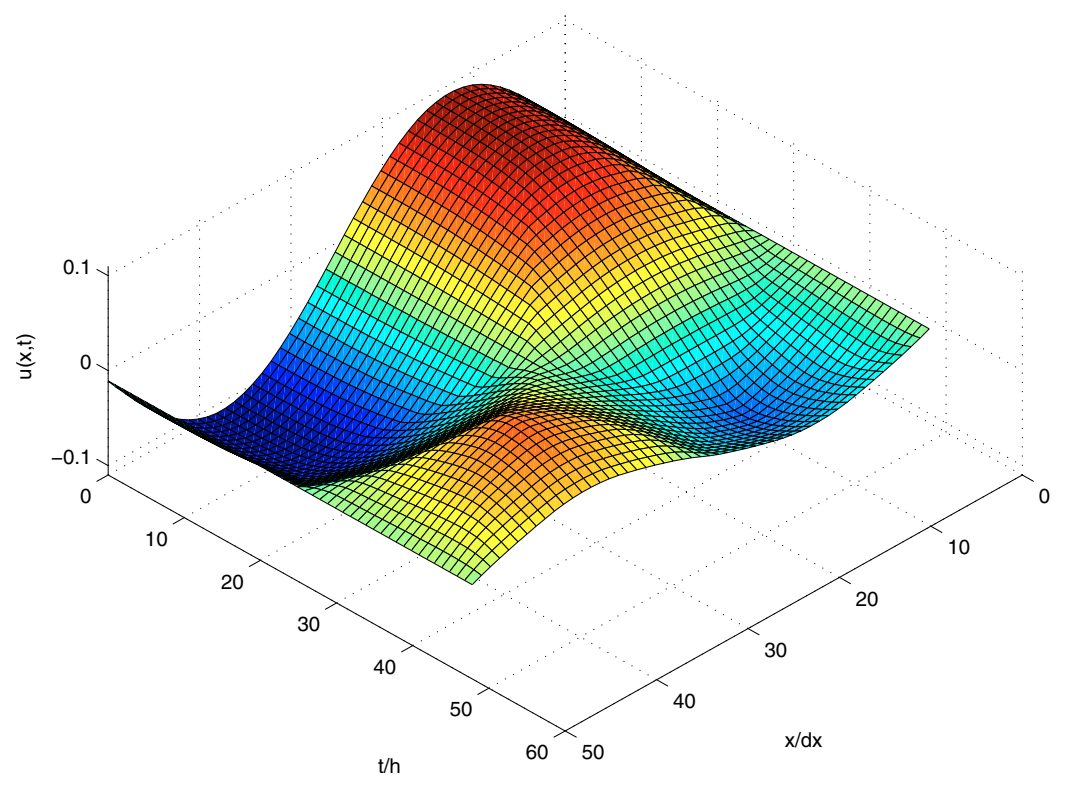

FiguRE 14. Numerical solution $u^{h, j}$ in Experiment $\mathrm{G}$ for $\mathrm{d} x=0.02$ and $h=0.02$ plotted over the space-time domain $(0,1) \times(0,1)$ in units of $t / h$ and $x / \mathrm{d} x$.

Experiment G. In the last experiment (see Fig. 14) we consider the inverse situation: We start with a deformed wire. Its deformation is stable below a certain temperature, but after passing this point it starts to oscillate. We choose the temperature as $\theta(t):=t-0.1$, and the initial conditions as $u_{0}(x):=0.1 \sin (\pi x), u_{1}=0$. There is essentially no change in the amplitude (unlike as in Experiment F). After passing the critical point, the wire starts to oscillate as if released from a deformed initial state.

\section{Extensions}

The condition $\phi^{q c}=\phi^{* *}$ of Theorem 2.1 is, in general, difficult to handle, since the quasiconvex envelope of a function is not easily accessible. A different characterization of this condition is given by the following result due to Kewei Zhang.

Theorem 6.1 [30]. If $\phi: \mathbb{R}^{m \times n} \rightarrow \mathbb{R}$ is a $\mathcal{C}^{1}$-function and has polynomial growth of order $p>1$ at infinity and if $\phi^{r c}$ denotes the rank-one-convexification of $\phi$, then

$$
\phi^{q c}=\phi^{* *} \Leftrightarrow \phi^{r c}=\phi^{* *} .
$$

For a given function $\phi$ the rank-one-convexification $\phi^{r c}$ can be calculated much easier than the quasiconvexification $\phi^{q c}$. Hence this theorem makes it feasible to test whether Theorem 2.1 can be applied for given energy densities:

Corollary 6.2. If $\phi$ is rank-one-convex but not convex, then it does not satisfy the assumptions of Theorem 2.1.

A similar result for a different time discretization scheme extends Theorem 2.1 and is in particular applicable if $\phi$ is quasiconvex. On the other hand it is not strictly more applicable than Theorem 2.1.

Theorem 6.3 (Existence). We define

$$
\tilde{\phi}\left(A_{1}, A_{2}\right):=\int_{0}^{1} \frac{1}{s}\left(\phi\left(s A_{1}+(1-s) A_{2}\right)-\phi\left(A_{2}\right)\right) \mathrm{d} s+\phi\left(A_{2}\right) .
$$




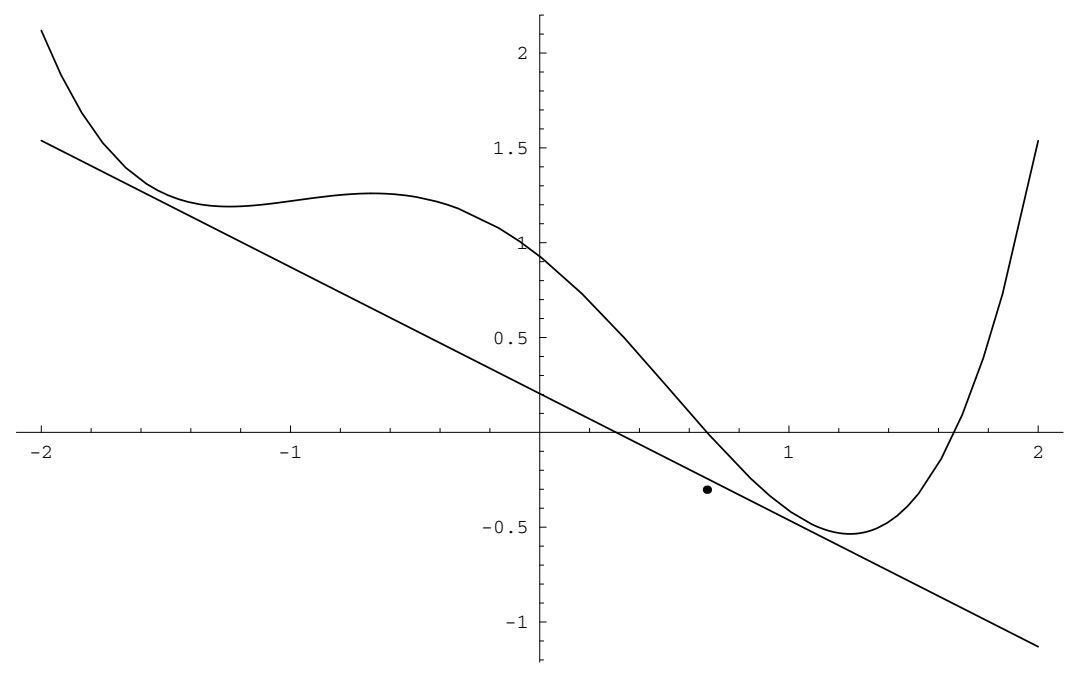

Figure 15. Plot of the line $l(A)$, the function $h(A)$ and the point $\left(A_{2},-\phi\left(A_{2}\right)\right)$ in the range $-2 \leq A \leq+2$. The line $l$ apparently separates $h$ and the point $\left(A_{2},-\phi\left(A_{2}\right)\right)$ (cf. Example 6.4).

Let $u_{0} \in H_{0}^{1}\left(\Omega ; \mathbb{R}^{m}\right), v_{0} \in L^{2}\left(\Omega ; \mathbb{R}^{m}\right)$ and

$$
\left.\tilde{\phi}^{q c}\left(A_{1}, A_{2}\right)\right|_{A_{1}=A_{2}}=\phi^{q c}\left(A_{1}\right) .
$$

Then there exists a Young-measure solution $(u, \nu)$ of our problem that can be obtained by the following time discretization scheme

$$
\begin{gathered}
\frac{u^{h, j}-2 u^{h, j-1}+u^{h, j-2}}{h^{2}}-\operatorname{div} \tilde{S}\left(D u^{h, j}, D u^{h, j-1}\right)=0, \\
u^{h, 0}=u_{0}, \quad u^{h,-1}=u_{0}-h v_{0},
\end{gathered}
$$

where

$$
\tilde{S}\left(A_{1}, A_{2}\right):=\int_{0}^{1} S\left(t A_{1}+(1-t) A_{2}\right) \mathrm{d} t .
$$

The discrete energy estimate can be proved for $\tilde{\phi}$ instead of $\phi$ without any convexity assumptions. The further existence proof follows closely the proof of Theorem 2.1 .

Condition (6.1) appears technical. However, it is needed for taking the final limit as the time-step size converges to zero. A sufficient condition for (6.1) is that the energy density $\phi$ is quasiconvex.

We might ask whether condition (6.1) holds for general energy functionals $\phi$, but this is not true: The following example shows that, even in simple non-quasiconvex situations, the envelope condition does not always hold.

Example 6.4. Consider $\phi(A):=\left(1-A^{2}\right)^{2}$. Set $A_{2}:=3 /(2 \sqrt{5})$ and $h\left(A_{1}\right):=\tilde{\phi}\left(A_{1}, A_{2}\right)-\phi\left(A_{2}\right)$. Then

$$
h\left(A_{1}\right)=\frac{1}{400}\left(\frac{1485}{4}-186 \sqrt{5} A_{1}-310 A_{1}^{2}+40 \sqrt{5} A_{1}^{3}+100 A_{1}^{4}\right) .
$$

A line $l$ separating the graph of $h$ from the point $\left(A_{2},-\phi\left(A_{2}\right)\right)$ is constructed as follows. Let $l$ be defined by the two points (see Fig. 15)

$$
\left(-\frac{3}{2}, h\left(-\frac{3}{2}\right)-\frac{5}{100}\right) \quad \text { and } \quad\left(1, h(1)-\frac{5}{100}\right)
$$


The line $l$ does not intersect the graph, and $l\left(A_{2}\right)>-\phi\left(A_{2}\right)$. Since $A_{2}<1$ and therefore $\phi^{q c}\left(A_{2}\right)=0$ we derive

$$
h^{q c}\left(A_{2}\right)+\phi\left(A_{2}\right) \geq h^{* *}\left(A_{2}\right)+\phi\left(A_{2}\right)>0=\phi^{q c}\left(A_{2}\right),
$$

and so

$$
\left.\tilde{\phi}^{q c}\left(A_{1}, A_{2}\right)\right|_{A_{1}=A_{2}}>\phi^{q c}\left(A_{2}\right) \text {. }
$$

Hence $\phi$ violates condition (6.1).

Acknowledgements. The research of CC was supported by the Max Planck Institute for Mathematics in the Sciences, Leipzig, Germany, the German Research Foundation through the DFG-Schwerpunktprogramm multi-scale problems, and the Isaac Newton Institute of Mathematical Sciences, Cambridge, UK; M.O.R. was supported by Max Planck Institute for Mathematics in the Sciences and by the Center for Nonlinear Analysis under NSF Grant DMS-9803791. He would like to thank Stefan Müller for his constant support and helpful suggestions.

\section{REFERENCES}

[1] J.M. Ball and R.D. James, Fine phase mixtures as minimizers of energy. Arch. Rational Mech. Anal. 100 (1987) 13-52.

[2] J.M. Ball, B. Kirchheim and J. Kristensen, Regularity of quasiconvex envelopes. Calc. Var. Partial Differential Equations 11 (2000) 333-359.

[3] H. Berliocchi and J.-M. Lasry, Intégrandes normales et mesures paramétrées en calcul des variations. Bull. Soc. Math. France 101 (1973) 129-184.

[4] C. Carstensen, Numerical analysis of microstructure, in Theory and numerics of differential equations (Durham, 2000), Universitext, Springer Verlag, Berlin (2001) 59-126.

[5] C. Carstensen and P. Plecháč, Numerical solution of the scalar double-well problem allowing microstructure. Math. Comp. 66 (1997) 997-1026.

[6] C. Carstensen and T. Roubíček, Numerical approximation of Young measures in non-convex variational problems. Numer. Math. 84 (2000) 395-415.

[7] C. Carstensen and G. Dolzmann, Time-space discretization of the nonlinear hyperbolic system $u_{t t}=\operatorname{div}\left(\sigma(D u)+D u_{t}\right)$. $S I A M$ J. Numer. Anal. 42 (2004) 75-89.

[8] M. Chipot, C. Collins and D. Kinderlehrer, Numerical analysis of oscillations in multiple well problems. Numer. Math. 70 (1995) 259-282.

[9] C. Collins and M. Luskin, Optimal-order error estimates for the finite element approximation of the solution of a nonconvex variational problem. Math. Comp. 57 (1991) 621-637.

[10] C. Collins, D. Kinderlehrer and M. Luskin, Numerical approximation of the solution of a variational problem with a double well potential. SIAM J. Numer. Anal. 28 (1991) 321-332.

[11] C.M. Dafermos and W.J. Hrusa, Energy methods for quasilinear hyperbolic initial-boundary value problems. Applications to elastodynamics. Arch. Rational Mech. Anal. 87 (1985) 267-292.

[12] S. Demoulini, Young-measure solutions for a nonlinear parabolic equation of forward-backward type. SIAM J. Math. Anal. 27 (1996) 376-403.

[13] S. Demoulini, Young-measure solutions for nonlinear evolutionary systems of mixed type. Ann. Inst. H. Poincaré Anal. Non Linéaire 14 (1997) 143-162.

[14] G. Friesecke and G. Dolzmann, Implicit time discretization and global existence for a quasi-linear evolution equation with nonconvex energy. SIAM J. Math. Anal. 28 (1997) 363-380.

[15] D. Kinderlehrer and P. Pedregal, Weak convergence of integrands and the Young measure representation. SIAM J. Math. Anal. 23 (1992) 1-19.

[16] P. Klouček and M. Luskin, The computation of the dynamics of the martensitic transformation. Contin. Mech. Thermodyn. 6 (1994) 209-240.

[17] M. Luskin, On the computation of crystalline microstructure, in Acta numerica, Cambridge Univ. Press, Cambridge (1996) $191-257$.

[18] S. Müller, Variational models for microstructure and phase transition, in Calculus of Variations and Geometric Evolution Problems, S. Hildebrandt and M. Struwe Eds., Lect. Notes Math. 1713, Springer-Verlag, Berlin (1999). 
[19] R.A. Nicolaides and N.J. Walkington, Computation of microstructure utilizing Young measure representations, in Transactions of the Tenth Army Conference on Applied Mathematics and Computing (West Point, NY, 1992), US Army Res. Office, Research Triangle Park, NC (1993) 57-68.

[20] P. Pedregal, Parametrized measures and variational principles. Birkhäuser (1997).

[21] M.O. Rieger, Time dependent Young measure solutions for an elasticity equation with diffusion, in International Conference on Differential Equations, Vol. 2 (Berlin, 1999), World Sci. Publishing, River Edge, NJ 1 (2000) $457-459$.

[22] M.O. Rieger, Young-measure solutions for nonconvex elastodynamics. SIAM J. Math. Anal. 34 (2003) 1380-1398.

[23] M.O. Rieger and J. Zimmer, Global existence for nonconvex thermoelasticity. Preprint 30/2002, Center for Nonlinear Analysis, Carnegie Mellon University, Pittsburgh, USA (2002).

[24] T. Roubíček, Relaxation in optimization theory and variational calculus. Walter de Gruyter \& Co., Berlin (1997).

[25] M. Slemrod, Dynamics of measured valued solutions to a backward-forward heat equation. J. Dynam. Differ. Equations 3 (1991) $1-28$.

[26] L. Tartar, Compensated compactness and applications to partial differential equations, in Nonlinear analysis and mechanics: Heriot-Watt Symposium. Pitman, Boston, Mass. IV (1979) 136-212.

[27] M.E. Taylor, Partial Differential Equations III. Appl. Math. Sciences. Springer-Verlag, 117 (1996).

[28] L.C. Young, Generalized curves and the existence of an attained absolute minimum in the calculus variations, volume classe III. (1937)

[29] L.C. Young, Lectures on the calculus of variations and optimal control theory. W.B. Saunders Co., Philadelphia (1969).

[30] K. Zhang, On some semiconvex envelopes. NoDEA. Nonlinear Differential Equations Appl. 9 (2002) 37-44.

To access this journal online: www.edpsciences.org 